Preprint

\title{
Reconnection in a Weakly Stochastic Field
}

\author{
A. Lazarian ${ }^{1,3}$ and Ethan T. Vishniac ${ }^{2}$ \\ ${ }^{1}$ Princeton University Observatory, Princeton, NJ 08544; lazarian@astro.princeton.edu \\ ${ }^{3}$ CITA, University of Toronto, Canada; lazarian@cita.utoronto.ca \\ ${ }^{2}$ Department of Physics and Astronomy, Johns Hopkins University, Baltimore MD \\ 21218; ethan@pha.jhu.edu
}

\begin{abstract}
We examine the effect of weak, small scale magnetic field structure on the rate of reconnection in a strongly magnetized plasma. This affects the rate of reconnection by reducing the transverse scale for reconnection flows, and by allowing many independent flux reconnection events to occur simultaneously. Allowing only for the first effect and using Goldreich and Sridhar's model of strong turbulence in a magnetized plasma with negligible intermittency, we find a lower limit for the reconnection speed $\sim V_{A} \mathcal{R}_{L}^{-3 / 16} \mathcal{M}^{3 / 4}$, where $V_{A}$ is the Alfvén speed, $\mathcal{R}_{L}$ is the Lundquist number, and $\mathcal{M}$ is the large scale magnetic Mach number of the turbulence. We derive an upper limit of $\sim V_{A} \mathcal{M}^{2}$ by invoking both effects. We argue that generic reconnection in turbulent plasmas will normally occur at close to this upper limit. The fraction of magnetic energy that goes directly into electron heating scales as $\mathcal{R}_{L}^{-2 / 5} \mathcal{M}^{8 / 5}$, and the thickness of the current sheet scales as $\mathcal{R}_{L}^{-3 / 5} \mathcal{M}^{-2 / 5}$. A significant fraction of the magnetic energy goes into high frequency Alfvén waves. The angle between adjacent field lines on the same side of the reconnection layer is $\sim \mathcal{R}_{L}^{-1 / 5} \mathcal{M}^{6 / 5}$ on the scale of the current sheet thickness. We claim that the qualitative sense of these conclusions, that reconnection is fast even though current sheets are narrow, is almost independent of the local physics of reconnection and the nature of the turbulent cascade. As the consequence of this the Galactic and Solar dynamos are generically fast, i.e. do not depend on the plasma resistivity.
\end{abstract}

Subject headings: Magnetic fields; Galaxies: magnetic fields, ISM: molecular clouds, magnetic fields 


\section{Introduction}

Magnetic reconnection is a long standing problem in theoretical magnetohydrodynamics (MHD). This problem is closely related to the hotly debated problem of the magnetic dynamo (see Parker 1979, Moffatt 1978, Krause \& Radler 1980). Indeed, it is impossible to understand the origin and evolution of large scale magnetic fields without a knowledge of the mobility and reconnection of magnetic fields. In a typical astrophysical plasma, resistivity is very small and flux freezing should be an excellent approximation to the motion of magnetic field, while dynamo action invokes a constantly changing magnetic field topology 1 This means an astrophysical dynamo requires efficient reconnection despite very slow Ohmic diffusion rates.

The literature on magnetic reconnection is rich and vast (see, for example, Biskamp 1993 and references therein). In the simplest model of magnetic reconnection (Parker 1957. Sweet 1958, see §3.1) the reconnection speed lies below the Alfvén speed by a factor $\sim \mathcal{R}_{L}^{-1 / 2}=\left(\eta / V_{A} L_{x}\right)^{1 / 2}$, where $\eta$ is the resistivity, $V_{A}$ is the Alfvén speed, and $L_{x}$ is the length of the current sheet, assumed to be determined by the large scale geometry of the problem. The large ratio of $L_{x}$ to the width of the current sheet, $\Delta$, equal to the ratio of the resistivity to the reconnection speed, drives the reconnection speed towards zero for highly conducting fluids. In particular, this is many orders of magnitude too small for astrophysical reconnection. Consequently, there has been intense interest in models which result in higher reconnection speeds.

In general, we can divide schemes for fast reconnection into those which alter the microscopic resistivity, broadening the current sheet, and those which change the global geometry, thereby reducing $L_{x}$. An example of the latter is the suggestion by Petschek (1964) that reconnecting magnetic fields would tend to form structures whose typical size in all directions is determined by the resistivity ('X-point' reconnection). This results in a reconnection speed of order $V_{A} / \ln \mathcal{R}_{L}$. On the other hand, attempts to produce such structures in simulations of reconnection have been disappointing (Biskamp 1984, Biskamp 1986). In such simulations the X-point region typically collapses towards the Sweet-Parker geometry as the Lundquist number becomes large (Biskamp 1996, Wang, Ma \& Bhattacharjee 1996, Wang \& Bhattacharjee 1996). One way to understand this collapse is to consider perturbations of the original $\mathrm{X}$-point geometry. In order to maintain this geometry reconnection has to be fast, which requires shocks in the original (Petschek)

\footnotetext{
${ }^{1}$ Merely winding up a magnetic field can increase the magnetic field energy, but cannot increase the magnetic field flux. We understand the dynamo in the latter sense. The Zel'dovich "fast" dynamo (Vainshtein \& Zel'dovich 1972) also invokes reconnection for continuous dynamo action (Vainshtein 1970).
} 
version of this model. These shocks are, in turn, supported by the flows driven by fast reconnection, and fade if $L_{x}$ increases. Naturally, the dynamical range for which the existence of such shocks is possible depends on the Lundquist number and shrinks when fluid conductivity increases. The apparent conclusion is that at least in the collisional regime reconnection occurs through narrow current sheets. [

In the collisionless regime the width of the current sheets may be determined by the ion cyclotron (or Larmor) radius (Parker 1979) or by the ion skin depth (Ma \& Bhattacharjee 1996, Biskamp, Schwarz, \& Drake 1997, Shay, Drake, Denton, \& Biskamp 1998). This often leads to a current sheet thickness which is much larger than expected ('anomalous resistivity'). Furthermore, anomalous resistivity can be combined with X-point reconnection with the result that the $\mathrm{X}$-point is somewhat less unstable to collapse. Nevertheless, while this effect can dramatically increase reconnection speeds in simulations and in laboratory experiments it does not change the qualitative conclusion that narrow current sheets give reconnection speeds in astrophysical plasmas that are unacceptably low. 3

In order to explain why astrophysical magnetic fields do not reverse on very small scales, researchers have usually appealed to an ad hoc diffusivity which is many orders of magnitude greater than Ohmic diffusivity. This diffusivity is assumed to be roughly equal to the local turbulent diffusion coefficient. While superficially reasonable, this choice implies that a dynamically significant magnetic field diffuses through a highly conducting plasma in much the same way as a tracer of no dynamical importance t. This is referred to as turbulent diffusivity and denoted $\eta_{t}$, as opposed to the Ohmic diffusivity $\eta$. Its name suggests that turbulent motions subject the field to kinematic swirling and mixing. As the field becomes intermittent and intermixed it can be assumed to undergo dissipation at arbitrarily high speeds.

Parker (1992) showed convincingly that the concept of turbulent diffusion is ill-founded. He pointed out that turbulent motions are strongly constrained by magnetic tension and large scale magnetic fields prevent hydrodynamic motions from mixing magnetic field

\footnotetext{
${ }^{2}$ A very different approach explored by Vishniac (1995a,b) appealed to the concentration of magnetic field in narrow flux tubes within a high $\beta$ plasma, thereby reducing $L_{x}$ to the flux tube radius. Given the small filling factor of the magnetic field and the high Alfven velocity inside the flux tubes it is possible to obtain fast reconnection even using the Sweet-Parker geometry. However, flux tube formation requires initially high reconnection rates.

${ }^{3}$ Reconnection in the presence of ambipolar diffusion discussed in Vishniac \& Lazarian (1998) vaguely fits into the category of "enhanced $\Delta$ ". Indeed, the neutrals do not feel magnetic field and leave the reconnection zone forming an outflow that is limited by ambipolar diffusion rather than Ohmic diffusivity.

${ }^{4}$ In the case of galaxies the ratio of the two diffusivities is $\sim 10^{20}$.
} 
regions of opposing polarity unless they are precisely anti-parallel. Both numerical and analytic studies (see Cattaneo \& Vainshtein 1991, Kulsrud \& Anderson 1992, Gruzinov \& Diamond 1994) confirm that the traditional (Ruzmaikin et al. 1988) theory of kinematic dynamos is seriously and fundamentally flawed. On the other hand, observations of the solar corona and chromosphere seem to show that reconnection often takes place at speeds of $\sim 0.1 V_{A}$ (cf. Dere 1996, Innes et al. 1997 and references contained therein). Evidently at least some astrophysical plasmas can undergo reconnection on short time scales, regardless of the apparent theoretical difficulties involved.

An associated problem for dynamo theories is the growth of small scale random magnetic fields. Assuming magnetic fields are allowed to intermix freely, as the turbulent diffusion model requires, Low (1972) and Krause \& Radler (1980) estimated that the ratio of the mean field to the random field is expected to be of the order $\sqrt{\eta_{t} / \eta} \gg 1$. At the same time, observations indicate that these field components are not very different in galaxies at the present epoch (Beck et al. 1996). Furthermore, Kulsrud and Anderson (1992) have suggested that the growth of a random field should suppress dynamo action.

This unsatisfactory state of affairs has stimulated recent attempts to find new approaches to the problem of the astrophysical dynamof (e.g. Parker 1992, Parker 1993, Vishniac 1995a,b, Kulsrud et al 1997) that have deficiencies which we will discuss in more detail below.

Below we explore a different approach to the problem of rapid reconnection. This paper deals with the issue of magnetic reconnection in the presence of a weak random field component. Magnetohydrodynamic turbulence guarantees the presence of such a field, although its amplitude and structure clearly depends on the model we adopt for MHD turbulence, as well as the specific environment of the field. It is also obvious from the above discussion that as long as the kinematic approximation holds, that is as long as magnetic forces are negligible, $\square$ the random component of the magnetic field will quickly come to dominate the large scale one. We will assume throughout that we can describe resistive

\footnotetext{
${ }^{5}$ The astrophysical dynamo is just an example of a process whose dynamics is determined by reconnection rates. Solar flaring (see Dere 1996) and star formation in molecular clouds (for example see Lubow \& Pringle 1996) are other examples.

${ }^{6}$ The mode of reconnection discussed here is sometimes is called free reconnection as opposed to forced reconnection. Wang \& Bhattacharjee (1992) define free reconnection as a process caused by a nonideal instability driven by the free energy stored in an equilibrium. If the equilibrium is stable, reconnection can be forced if a perturbation is applied externally.

${ }^{7}$ This approximation should be valid, at least, at the initial stages of magnetic field generation.
} 
effects with an Ohmic resistivity $\eta$. However, our qualitative conclusions are not sensitive to this choice. We will show that if we allow for the more complicated geometry expected from a turbulent cascade, the speed of reconnection is fast, independent of the plasma resistivity, and a function only of the overall level of turbulence. Although we will adopt a specific model of MHD turbulence in this work, we will also show that our conclusions are not sensitive to this choice.

There are two mechanisms which lead to this result. First, if we consider two adjoining regions with very different large scale fields, the small scale structure of both fields implies that the typical scale for a reconnection event is much smaller than the overall size of the system, and goes to zero in the limit of an ideal fluid. Second, since field lines wander in and out of the reconnection zone, the overall rate of flux reconnection can be much greater than the rate for a single event. We note that it is usually believed that for reconnection to be rapid in the limit of $\eta \rightarrow 0$ a current singularity should develop (Park et al 1984). Our model does not require such singularities. Indeed, we show that while the amount of Ohmic dissipation tends to 0 as $\eta \rightarrow 0$, the magnetic field has a weak stochastic substructure on the smallest scales and the rate of the flux reconnection does not decrease.

In $\S 2$ of this paper we will introduce the scheme of reconnection in the presence of stochastic field, while in $\S 3$ of this paper we will describe the results of a simple model for the small scale structure of a large scale magnetic field embedded in a turbulent medium. A fuller discussion of this model is given in Appendix A. In $\S 4$ we will estimate the reconnection speed in the presence of small amounts of stochasticity, and apply these

estimates to the model described in $\S 3$. In $\S 5$ we describe some of the implications of this work. We give our conclusions in $\S 6$.

\section{The Problem}

Sweet-Parker reconnection (Sweet 1958, Parker 1957) provides a minimum value for reconnection rates. In this model oppositely directed magnetic fields are brought into contact over a region of size $L_{x}$. Magnetic fields reconnect along a very thin Ohmic diffusion layer $L_{y} \approx \eta / V_{\text {rec }}$ and fluid is ejected from this layer at a velocity of the order $V_{A}$ in a direction parallel to the local field lines. The layer in which Ohmic diffusion takes place is usually referred to as the current sheet. Here we will refer to the volume where the mean magnetic field strength drops significantly as the reconnection zone, in order to allow for the presence of collective effects which may broaden the reconnection zone well beyond the current sheet. The reconnection velocity in the Sweet-Parker picture is determined by the constraint imposed by the conservation of mass condition $V_{r e c} L_{x} \approx V_{A} L_{y}$. Although 
this model is two dimensional, it can be generalized to three dimensions by allowing the two magnetic field regions to share a common field component, which has the effect of rotating them so that they are no longer exactly anti-parallel. This has no effect on the Sweet-Parker reconnection process (see Fig. 1). However, it does change the nature of the constraint somewhat. In addition to ejecting matter from the reconnection zone, we must also allow for the ejection of the magnetic flux due to the common field component. This is, in effect, the same constraint in this case.

We consider the case in which there exists a large scale, well-ordered magnetic field, of the kind that is normally used as a starting point for discussions of reconnection. This field may, or may not, be ordered on the largest conceivable scales. However, we will consider scales smaller than the typical radius of curvature of the magnetic field lines, or alternatively, scales below the peak in the power spectrum of the magnetic field, so that the direction of the unperturbed magnetic field is a reasonably well defined concept. In addition, we expect that the field has some small scale 'wandering' of the field lines. On any given scale the typical angle by which field lines differ from their neighbors is $\phi \ll 1$, and this angle persists for a distance along the field lines $\lambda_{\|}$with a correlation distance $\lambda_{\perp}$ across field lines.

The modification of the mass conservation constraint in the presence of stochastic magnetic field component is self-evident. Instead of being squeezed from a layer whose width is determined by Ohmic diffusion, the plasma may diffuse through a much broader layer, $L_{y} \sim\left\langle y^{2}\right\rangle^{1 / 2}$ (see Fig. 2), determined by the diffusion of magnetic field lines. The value of $\left\langle y^{2}\right\rangle^{1 / 2}$ can be determined once a particular model of turbulence is adopted (see $\S 3$ ), but it is obvious from the very beginning that this value is determined by field wandering rather than Ohmic diffusion as in the Sweet-Parker case.

In the presence of a stochastic field component, magnetic reconnection dissipates field lines not over their entire length $\sim L_{x}$ but only over a scale $\lambda_{\|} \ll L_{x}$ (see Fig. 2b), which is the scale over which magnetic field line deviates from its original direction by the thickness of the Ohmic diffusion layer $\lambda_{\perp}^{-1} \approx \eta / V_{\text {rec,local }}$. If the angle $\phi$ of field deviation does not depend on the scale, the local reconnection velocity would be $\sim V_{A} \phi$ and would not depend on resistivity. We claim in $\S 3$ that $\phi$ does depend on scale. Therefore the local reconnection rate $V_{\text {rec,local }}$ is given by the usual Sweet-Parker formulae but with $\lambda_{\|}$instead of $L_{x}$, i.e. $V_{\text {rec,local }} \approx V_{A}\left(V_{A} \lambda_{\|} / \eta\right)^{-1 / 2}$. It is obvious from Fig. 2a that $\sim L_{x} / \lambda_{\|}$magnetic field lines will undergo reconnection simultaneously (compared to a one by one line reconnection process for the Sweet-Parker scheme). Therefore the overall reconnection rate may be as large as $V_{\text {rec,global }} \approx V_{A}\left(L_{x} / \lambda_{\|}\right)\left(V_{A} \lambda_{\|} / \eta\right)^{-1 / 2}$, which means that the reconnection efficiency critically depends on the value of $\lambda_{\|}$. More realistically we will find that there are other global 
constraints which end up determining the actual global reconnection speed.

The relevant values of $\lambda_{\|}$and $\left\langle y^{2}\right\rangle^{1 / 2}$ depend critically on the magnetic field statistics. Therefore in the next section we will briefly explore the expected properties of magnetic turbulence.

\section{Magnetic Field Structure in a Turbulent Medium}

The study of MHD turbulence is hampered by the absence of easily observable examples. We will base our discussion here on a model first proposed by Goldreich and Sridhar (1995, hereinafter GS95), which is at least consistent with available observations of the interstellar medium and the solar wind. A discussion of this model, and the justification for using it as a first approximation is given in Appendix A. In any case, the qualitative nature of our results, that a weak stochastic component to the field structure can have a dramatic effect on reconnection rates, is not sensitive to the details of the model we adopt. We discuss reconnection for various power spectra including the Kraichnan spectrum (Iroshnikov 1963, Kraichnan 1965) in Appendix D.

For strong turbulence we have $\lambda_{\perp} \approx \lambda_{\|} \phi\left(\lambda_{\|}\right)$, although we can also define a weakly turbulent regime where the correlation length is much greater than the distance by which individual field lines deviate from a straight line. In either case, we can define a corresponding perpendicular wavenumber, $k_{\perp} \sim \lambda_{\perp}^{-1}\left(\lambda_{\|}\right)$.

In order to describe this wandering, we need to know something about quasi-static distortions of the field lines, or 'zero-frequency' modes. Normally, this means only those distortions of the magnetic field whose evolution is driven primarily by nonlinear effects. Here, however, we mean simply all modes that evolve on time scales long enough that they can be considered as a background to the evolution of flows driven by reconnection. Motions that can be described as Alfvén waves may be relevant in this sense, as long as they involve very long wavelengths parallel to the magnetic field. However, most of our discussion will be concerned with modes with nonlinear time scales comparable to their wave periods, so that they are Alfvén waves only in a very general sense. For simplicity,

we will consider incompressible turbulence and assume that the magnetic field filling factor is not too small. The former should be a reasonable approximation for small scale, and therefore weak, fluid motions. The latter is probably a bad approximation inside the Sun, although reasonable whenever the magnetic pressure is comparable to, or greater than, the gas pressure (or when intermittency has not had time to develop).

We can describe a model of MHD turbulence in terms of the relationship between 
$k_{\perp}$ and $k_{\|}$at every scale in the turbulent cascade, as well as the scaling of $v_{k}$ as function of wavenumber. By restricting ourselves to a model in which field stochasticity is driven locally by a turbulent cascade we are neglecting the possibility that the field lines are anchored in some denser medium, like field lines extruded from the photosphere of the Sun, but even in this case the field lines are mixed at their footpoints by turbulent motions in the photosphere (see, for example, Parker 1988, and references contained therein). More generally there will be cases in which the level of stochasticity will be determined by nonlocal processes. Here we will restrict ourselves to cases where the field stochasticity can be understood in terms of local turbulence.

If energy is injected on some scale $l$, with $v_{l} \leq V_{A}$, then GS95 predict that

$$
\begin{gathered}
k_{\|} \approx l^{-1}\left(k_{\perp} l\right)^{2 / 3}\left(\frac{v_{l}}{V_{A}}\right)^{4 / 3}, \\
\tau_{n l}^{-1} \approx \frac{v_{l}}{l}\left(k_{\perp} l\right)^{2 / 3}\left(\frac{v_{l}}{V_{A}}\right)^{1 / 3},
\end{gathered}
$$

while the rms fluid velocity is given by

$$
v_{k} \approx v_{l}\left(k_{\perp} l\right)^{-1 / 3}\left(\frac{v_{l}}{V_{A}}\right)^{1 / 3} .
$$

These equations are derived in Appendix A. Equation (11) is particularly interesting. It gives the geometry of small scale magnetic field structure in a turbulent cascade. This turns out to be critically important in magnetic reconnection.

A bundle of field lines confined within a region of width $y$ at some particular point will spread out perpendicular to the mean magnetic field direction as one moves in either direction parallel to the local magnetic field lines. The rate of field line diffusion is given approximately by

$$
\frac{d\left\langle y^{2}\right\rangle}{d x} \sim \frac{\left\langle y^{2}\right\rangle}{\lambda_{\|}}
$$

where $\lambda_{\|}^{-1} \approx k_{\|}, k_{\|}$is the parallel wavevector chosen so that the corresponding vertical wavelength, $k_{\perp}\left(k_{\perp}\right)$, is $\sim\left\langle y^{2}\right\rangle^{1 / 2}$, and $x$ is the distance along an axis parallel to the mean magnetic field. Therefore, using equation (11) one gets

$$
\frac{d\left\langle y^{2}\right\rangle}{d x} \sim l\left(\frac{\left\langle y^{2}\right\rangle}{l^{2}}\right)^{2 / 3}\left(\frac{v_{l}}{V_{A}}\right)^{4 / 3}
$$

where we have substituted $\left\langle y^{2}\right\rangle^{-1 / 2}$ for $k_{\perp}$. This expression for the diffusion coefficient will only apply when $y$ is small enough for us to use the strong turbulence scaling relations, 
or in other words when $\left\langle y^{2}\right\rangle<l^{2}\left(v_{l} / V_{A}\right)^{4}$. Larger bundles will diffuse at a maximum rate of $l\left(v_{l} / V_{A}\right)^{4}$. For $\left\langle y^{2}\right\rangle$ small equation (5) implies that a given field line will wander perpendicular to the mean field line direction by an average amount

$$
\left\langle y^{2}\right\rangle^{1 / 2}=\frac{(3 x)^{3 / 2}}{l^{1 / 2}}\left(\frac{v_{l}}{V_{A}}\right)^{2}
$$

in a distance $x$. The fact that the rms perpendicular displacement grows faster than $x$ is significant. It implies that if we consider a reconnection zone, a given magnetic flux element that wanders out of the zone has only a small probability of wandering back into it.

\section{Turbulent Reconnection}

\subsection{Constraints on reconnection rate}

Outflow of matter from the reconnection layer constrains the achievable reconnection rates. In the presence of turbulence the thickness of the outflow layer increases with $L_{x}$ according to equation (6):

$$
\left\langle y^{2}\right\rangle^{1 / 2} \sim L_{x}\left(\frac{L_{x}}{l}\right)^{1 / 2}\left(\frac{v_{l}}{V_{A}}\right)^{2}
$$

when $l>L_{x}$ and

$$
\left\langle y^{2}\right\rangle^{1 / 2} \sim\left(L_{x} l\right)^{1 / 2}\left(\frac{v_{l}}{V_{A}}\right)^{2}
$$

when $L_{x}>l$. Therefore the upper limit on $V_{\text {rec }}$ imposed by large scale field line diffusion is

$$
V_{\text {rec }}<V_{A} \min \left[\left(\frac{L_{x}}{l}\right)^{1 / 2},\left(\frac{l}{L_{x}}\right)^{1 / 2}\right]\left(\frac{v_{l}}{V_{A}}\right)^{2} .
$$

This limit on the reconnection speed is fast, both in the sense that it does not depend on the resistivity, and in the sense that it represents a large fraction of the Alfvén speed. Whether or not this limit is attainable depends to a large extent on the motion of the shared component of the magnetic flux. Nevertheless, we shall see below that equation (9) is not merely an upper limit on the global reconnection speed, but under most circumstances, constitutes a reasonable estimate for its actual value.

There are two important issues that we have neglected in arriving at this estimate. First, we have assumed an outflow velocity of $V_{A}$ spread over the entire width of the outflow region. However, in general the field lines that pass through the reconnection zone at any one moment will not fill the entire width of the outflow region. Instead, they will fill some fraction of it, with a proportionate drop in the effective outflow velocity. This reduction 
will be almost exactly offset by the fact that every flux element stays in the reconnection zone for roughly $L_{x} / V_{A}$ and that defines the meaning of "simultaneous" in this case. When reconnection starts, the outflow fills only a small fraction of the reconnection layer and this corresponds to the minimal estimate of the reconnection rate below. However, as reconnection proceeds, more independent flux elements become involved and this enhances the rate.

In addition, equation (9) is based on the assumption that the field line topology far from the reconnection layer is not affected by the process of reconnection. In reality we expect that, once reconnection begins, the changing field line stresses will be communicated throughout the volume at a speed $V_{A}$. Also, the reconnection layer will act as a source of small scale nonlinear Alfvén waves. The outcome of these effects is somewhat uncertain, but it will probably have the effect of accelerating reconnection.

We can obtain a lower limit on the reconnection speed by narrowing our focus and remembering that plasma can escape from the reconnection layer proper only by traveling a distance $\lambda_{\|}\left(k_{\perp}\right)$ along the local field lines, where $k_{\perp}^{-1} \sim \Delta$ is the thickness of the reconnection layer. A lower limit on the reconnection speed follows from the mass conservation condition:

$$
V_{\text {rec,local }} \lambda_{\|} \approx V_{A} \Delta
$$

and the requirement that at a fundamental level reconnection is driven by Ohmic diffusion:

$$
V_{\text {rec,local }} \approx \eta / \Delta
$$

We have denoted this estimate for the reconnection speed with a special subscript not only because its value is determined by local physics, but also because we shall see that it is likely that the global speed of reconnection through a field line bundle is different, and much faster, than this local value. Combining equations (1), (10) and (11) and recalling that $k_{\perp}^{-1} \sim \Delta$ and $k_{\|}^{-1} \sim \lambda_{\|}$we get reconnection at a speed

$$
V_{\text {rec,local }} \sim \frac{V_{A}}{k_{\perp} \lambda_{\|}} \sim V_{A}\left(k_{\perp} l\right)^{-1 / 3}\left(\frac{v_{l}}{V_{A}}\right)^{4 / 3} \sim V_{A}\left(\frac{V_{\text {rec,local }} l}{\eta}\right)^{-1 / 3}\left(\frac{v_{l}}{V_{A}}\right)^{4 / 3}
$$

which implies that

$$
V_{\text {rec }, \text { local }} \sim v_{l}\left(\frac{\eta}{V_{A} l}\right)^{1 / 4}
$$

In arriving at this estimate we have treated field perturbations with frequencies less than $\sim V_{A} / \lambda_{\|}$as essentially static. However, since we see from equations (10) and (11) that the reconnection rate $\eta / \Delta^{2}$ is $\sim V_{A} / \lambda_{\|}$, this is a reasonable approximation. Finally, we note 
that this process is controlled by field fluctuations with a perpendicular wavenumber

$$
k_{\perp} \approx \frac{V_{r e c, l o c a l}}{\eta} \approx l^{-1} \frac{v_{l}}{V_{A}}\left(\frac{V_{A} l}{\eta}\right)^{3 / 4} .
$$

We see from equation (A11) that this is the largest wavenumber we can expect in the turbulent cascade, or at least the largest we can expect before the reconnection process generates its own local turbulence.

If, however, the medium is partially ionized the maximum wavenumber given in equation (A11) should be reduced by a factor $\sim\left(\eta / \eta_{a m b}\right)^{3 / 4}$. This will reduce the reconnection speed by a factor of $\sim\left(\eta / \eta_{a m b}\right)^{1 / 4}$, but this reduction will be somewhat offset by the fact that the ions will be compressed until their pressure alone is comparable to the magnetic field pressure, and it is this compressed layer of ions which is ejected from the reconnection region along the field lines. The resulting velocity should be compared with the ambipolar reconnection velocity from (Vishniac \& Lazarian 1998):

$$
V_{\text {ambipolar }}=\left(\frac{2 \eta}{\tau_{\text {recomb }}}\right)^{1 / 2} \frac{1}{\beta X}(1+2 \beta x)^{1 / 2}
$$

whether $X$ is the ionization ratio, $\beta$ is the ratio of the gas pressure to the magnetic pressure. The recombination time of the ions $\tau_{\text {recomb }}$ as well as Ohmic diffusivity $\eta$ are taken far gas from the reconnection layer. The larger of the two velocities will determine the reconnection speed. Results in Table 1 in Vishniac \& Lazarian (1998) show that the reconnection speed can be increased via ambipolar diffusion by a factor of $10^{3}$, which is not much, if we account for the fact that the original Sweet-Parker rate is very slow. A detailed account of reconnection in the presence of MHD turbulence and ambipolar diffusion is given in Lazarian \& Vishniac (1998).

So far we have ignored the fact that the thin current sheets envisioned in Sweet-Parker reconnection are unstable. We briefly address this issue in Appendix $\mathrm{C}$ and show that allowing for the presence of tearing modes raises the minimum value of $V_{\text {rec,local }}$ to $\sim V_{A}\left(\frac{v_{l}}{V_{A}}\right)^{3 / 4}\left(\frac{\eta}{V_{A} l}\right)^{3 / 16}$. Even in the context of large scale turbulence in the interstellar medium, this is only an increase of about one order of magnitude, and still leaves us with $V_{\text {rec,local }} \ll V_{A}$.

\subsection{Estimating the global value of $V_{r e c}$}

The physics of the reconnection layer will be significantly more complicated than the simple picture above indicates. First of all, a number of known processes can enhance 
reconnection. In the appendices we discuss Bohm diffusion, anomalous resistivity and tearing modes. All of these have been discussed previously as ways to accelerate reconnection, but none have emerged as universal mechanisms for producing fast reconnection. In a previous paper (Vishniac and Lazarian 1998) we have shown that while ambipolar diffusion can produce dramatically faster reconnection rates, the overall pace of reconnection is still orders of magnitude below $V_{A}$. Anomalous resistivity (see Appendix B) is of marginal importance for interstellar reconnection, although it can be important for laboratory plasma. Finally, Bohm diffusion (Appendix B) can provide a strong enhancement of reconnection speeds, but unfortunately it is unclear whether or not the empirical concept of Bohm diffusion is applicable to astrophysical plasmas.

All of this is probably less important than the global ordering of reconnection events. The minimal estimate of $V_{\text {rec }}$ given in the previous subsection is based on the assumption that reconnection proceeds sequentially, that is, the reconnection speed is simply the speed with which reconnection propagates through a single flux element. This is not obviously correct, since the reconnection zone contains many independent reconnection events at any one time. We need to define a global reconnection speed, $V_{\text {rec,global }}$, which describes the rate at which flux is reconnected throughout the reconnection zone. In order to arrive at a reasonable estimate of this speed, we have to determine which aspect of reconnection sets a limit on its efficiency. Based on our previous discussion, we can see that there are four possibilities: the mass flow from the reconnection zone itself, the speed with which reconnected flux elements move across the reconnection zone and off the edge, the ejection of the flux associated with the shared magnetic field component, and the mass flow from the contact volume (roughly everything within a distance $L_{x}$ of the reconnection zone). In the case of Sweet-Parker reconnection the first process provides the critical constraint (and the third and fourth are not separate constraints).

The first limit is determined by $V_{\text {rec,local }}$, but is not equal to it. Since the current sheet contains as many as $k_{\perp} k_{\|} L_{x}^{2}$ independent reconnection surfaces, each one reconnecting flux at a rate $\lambda_{\perp}^{-1} V_{\text {rec,local }}$, the global reconnection speed could be enhanced by a factor $\left.\right|^{\natural}$ of $k_{\|} L_{x}$. In other words, using equations (1), (13), and (14) we get

$$
V_{\text {rec,global }}=k_{\|} L_{x} V_{\text {rec }, \text { local }}=V_{A}\left(\frac{V_{A} l}{\eta}\right)^{1 / 4} \frac{L_{x}}{l}\left(\frac{v_{l}}{V_{A}}\right)^{3} .
$$

Since this will almost always be less restrictive than equation (9), we conclude that the flow

\footnotetext{
8 The ambipolar reconnection rate given by Eq. (15) is a local reconnection speed. Therefore, in the presence of turbulence the global ambipolar reconnection speed is larger by a similar factor (Lazarian \& Vishniac 1999).
} 
of matter from the reconnection zone itself does not limit the speed of reconnection.

The second limit is set by the speed with which reconnected magnetic field elements can pass through one another on their way to the edge of the reconnection zone. After initial reconnection magnetic field elements will move until they encounter magnetic flux moving in the opposite direction. If this process limits the speed of reconnection, then we can assume that each flux element has a typical size comparable to the width of the reconnection zone, and moves a similar distance before being entangled by another flux element. (If the reconnected flux elements were distributed more sparsely, then from the preceding paragraph we can see that the gaps would fill in with freshly reconnected flux.) We can restrict ourselves to the case where the flux elements cross the reconnection layer vertically, rather than at a shallow angle, since in the latter case reconnection between opposing flux surfaces would, if anything, be enhanced and the volume filled by a single reconnected element would evolve into one filled by many flux elements with a nearly vertical orientation. Outside the reconnection layer the magnetic field moves parallel to the reconnection layer only a small amount, and reverses direction after each interchange. The layers of magnetic field around the reconnection zone are removed starting from its edges and working inwards.

The steady pace of reconnection ensures that the reconnection zone is in a constant state of turbulent motion. In the absence of collisions between flux elements we could assume an average transverse velocity of $V_{A}$, but in their presence we need to estimate the reconnection speed for these flux elements. In an important sense, this is just a repetition of the problem we face in finding $V_{\text {rec,global }}$. The only difference is that turbulent motions within the reconnection zone guarantee a local source of turbulence. The level of turbulence in a reconnection layer of width $\Delta$ is given by equating the energy injection rate, $V_{A}^{2}\left(V_{\text {eject }} / \Delta\right)$, with the turbulent dissipation rate, $\sim v_{\text {local }}^{4} / V_{A} \Delta$. We conclude that

$$
v_{\text {local }} \sim V_{A}^{3 / 4} V_{\text {eject }}^{1 / 4}
$$

We note that the limiting value of $k_{\perp}$ imposed by dissipation outside the reconnection zone will be replaced locally by some much larger value due to this separate source of turbulence. Consequently, if the motion of flux elements in the reconnection zone limit the global pace of reconnection, and if we adopt the ansatz's that

$$
V_{\text {rec,global }}=V_{A}\left(\frac{\eta}{l V_{A}}\right)^{n}\left(\frac{v_{l}}{V_{A}}\right)^{m}
$$

\footnotetext{
${ }^{9}$ We do not consider the possibility of a logarithmic dependence on the Lundquist number, as in this case reconnection is already fast.
} 
where $m$ and $n$ are to be determined, then the average ejection speed of magnetic flux from the sides of the reconnection zone is just

$$
V_{\text {eject }}=V_{A}\left(\frac{\eta}{\Delta V_{A}}\right)^{n}\left(\frac{V_{\text {eject }}}{V_{A}}\right)^{m / 4} .
$$

On the other hand, if reconnected magnetic flux elements fill a large fraction of the reconnection zone, as they must if they determine the overall pace of reconnection, then

$$
V_{\text {rec,global }} \approx V_{\text {eject }} .
$$

We see from equations $(\sqrt{19})$ and $(20)$ that

$$
V_{\text {rec,global }} \approx V_{A}\left(\frac{\eta}{\Delta V_{A}}\right)^{\frac{n}{1-(m / 4)}}
$$

We can see from this that $m<4$, but there is no obvious constraint on $n$. However, since the intersection of separate flux elements constitutes a repetition, on a smaller scale, of the global process of reconnection, we expect to find a still smaller scale $\Delta^{\prime}$ which characterizes the size of the flux elements generated during the reconnection of flux elements of size $\Delta$. The same arguments then allow us to conclude that

$$
V_{\text {rec,global }} \approx V_{A}\left(\frac{\eta}{\Delta^{\prime} V_{A}}\right)^{\frac{n}{1-(m / 4)}}
$$

From equations (21) and (22) we see that $n=0$, and (now referring to equations (18) and (22)) that $m=0$ as well. In other words, adopting the hypothesis that reconnection is limited by rate of interchange of the tangle of reconnected flux elements that cross the reconnection layer we conclude only that $V_{\text {rec,global }} \lesssim V_{A}$. Since this limit is less stringent than equation (9) we see that this process is not, in fact, the bottleneck for turbulent reconnection and may therefore be ignored.

Our argument assumes that the scale $\Delta$ is greater than the spectrum cutoff, $k_{\perp, \text { max }}^{-1}$, for the external turbulence and that $\Delta^{\prime}$ is similarly greater than the local spectrum cutoff $k_{\perp, \text { max }}^{\prime}$. We can check this by adopting the very conservative constraint that the flux element widths are greater than the scale imposed by Ohmic diffusion with the time necessary for a reconnection front to propagate over the reconnection layer, that is

$$
\Delta>\left(\frac{\eta L_{x}}{V_{\text {rec, }, \text { lobal }}}\right)^{1 / 2} \approx\left(\frac{\eta}{L_{x} V_{A}}\right)^{1 / 2} L_{x}\left(\frac{V_{A}}{V_{\text {rec }, \text { global }}}\right)^{1 / 2}
$$

and

$$
\Delta^{\prime}>\left(\frac{\eta \Delta}{V_{\text {rec, global }}}\right)^{1 / 2}
$$


or

$$
\frac{\Delta^{\prime}}{\Delta}>\left(\frac{\eta}{\Delta V_{\text {rec,global }}}\right)^{1 / 2}
$$

From equation (A11) we have

$$
k_{\perp, \max } \Delta>\left(\frac{v_{l} L_{x}}{\eta}\right)^{1 / 4}\left(\frac{v_{l}}{V_{\text {rec,global }}}\right)^{1 / 4}\left(\frac{V_{A}}{V_{\text {rec, }, \text { lobal }}}\right)^{1 / 4}\left(\frac{L_{x}}{l}\right)^{1 / 4} .
$$

Since all these factors, except possibly the last, are larger than one, and the first is usually much larger than one, we can conclude that $k_{\perp, \max } \Delta>1$. Similarly, the local turbulence gives us a modified limit on $k_{\perp}$ which is

$$
k_{\perp, \text { max }}^{\prime} \sim \Delta^{-1}\left(\frac{V_{r e c, g l o b a l}}{V_{A}}\right)^{1 / 4}\left(\frac{V_{A} \Delta}{\eta}\right)^{3 / 4}
$$

Combining this with equation (25) we have

$$
k_{\perp, \text { max }}^{\prime} \Delta^{\prime}>\left(\frac{\eta}{\Delta V_{\text {rec }, \text { global }}}\right)^{1 / 2}\left(\frac{V_{A} \Delta}{\eta}\right)^{3 / 4}\left(\frac{V_{\text {rec }, \text { global }}}{V_{A}}\right)^{1 / 4}>\left(\frac{V_{A} \Delta}{\eta}\right)^{1 / 4}\left(\frac{V_{A}}{V_{\text {rec }, \text { global }}}\right)^{1 / 4},
$$

which is strictly greater than one. We conclude that the our assumption of a self-similar hierarchy is self-consistent.

We note that the fact that the horizontal transport of flux elements does not provide the limiting constraint on $V_{\text {rec,global }}$ implies that the fraction of the reconnection zone filled with already reconnected flux elements, $f_{r}$, is not necessarily large. However, there is firm lower limit on $f_{r}$. Since $V_{\text {rec,global }}=f_{r} V_{\text {eject }}$ then since $V_{\text {eject }} \leq V_{A}$,

$$
f_{r}>\frac{V_{\text {rec,global }}}{V_{A}} .
$$

If reconnection is fast then $f_{r}$ must be of order unity.

The third limit involves the width of the zone through which the shared component of the magnetic field is ejected, $w_{\text {eject }}$. Given the stochastic geometry of the initial field configuration, and its evolution during reconnection, this is not necessarily the same as the width of the reconnection zone. In any case we have

$$
V_{\text {rec }, \text { global }} L_{x}=V_{\text {eject }} w_{\text {eject }}=w \frac{V_{r e c, g l o b a l}}{f_{r}}
$$

or

$$
w_{\text {eject }}=f_{r} L_{x} .
$$


In a certain sense, this is just a restatement of the hypothesis that the ejection of the shared flux from the contact volume controls the speed of reconnection. However, there is another connection between $w_{\text {eject }}$ and $f_{r}$ where causality runs in the other direction. If we consider the initial surface layer of flux elements facing the reconnection zone, they have connections which run deep into the underlying magnetized region (cf. equations (7) and (8)). However, these connections have a small volume filling factor. Once these initial elements reconnect, further reconnection involves flux elements that are pressed down between the reconnected elements. This gives them an extra component normal to the plane of the reconnection zone, which we can express as a displacement of the flux element from its initial position. As $f_{r} \rightarrow 1$ (or at least a fraction of order unity) this displacement is typically as large as the diffusion limit given by equations (7) and (8) and the volume filling factor of the reconnected flux elements rises to one. Since typical flux elements will acquire a vertical stretch equal to this displacement, we see that this will also be the width of the escape zone for the shared flux. In other words, if reconnected flux elements fill up the reconnection zone, then $w_{\text {eject }}$ will be of order $f_{r}$ and shared flux can escape without any difficulty.

Is this a likely scenario? If the reconnection zone is sparsely filled, then nearby flux elements will be forced into it by the local pressure excess. Since matter can escape without difficulty in this model, this pressure excess is equal to the magnetic pressure. This will not be sufficient to force magnetic field lines to bend at sharp angles, unless the whole excess is supported on a few field lines. However, it will be sufficient to force nearly parallel field lines to move, so we can envision this motion as consisting almost entirely of motion towards the reconnection zone, without any significant bending away from the initial mean field direction parallel to the reconnection zone. Furthermore, as long as $w_{\text {eject }}$ is no larger than the value of $k_{\perp}^{-1}$ corresponding to parallel wavelengths $\sim L_{x}$, then the turbulent motions within the magnetized fluid can be assumed to keep moving flux elements up to the reconnection zone. This will ensure that the reconnection zone is continuously supplied with magnetic elements to reconnect, and consequently the $f_{r}$ will grow until the loss of magnetic flux from the reconnection zone balances the supply. This implies that $w_{\text {eject }}$ will grow until some other process limits the global speed of reconnection.

The fourth, and last, limit is equation (9). We see from the three preceding arguments that this is not just an upper limit, but a reasonable estimate for the global speed of reconnection in a turbulent medium. Reconnection will generally occur at a substantial fraction of the Alfvén speed with the level of turbulence in the medium (or alternatively, the level of stochasticity in the magnetic field) controlling the exact speed.

We can turn this line of reasoning around, and use the global speed of reconnection to estimate the properties of the reconnection zone itself. First, we note that the field lines 
will bend down to the reconnection zone with a typical displacement equal to the diffusion distance, or for $L_{x}<l$,

$$
w_{\text {eject }}=\left(\frac{v_{l}}{V_{A}}\right)^{2} \frac{L_{x}^{3 / 2}}{l^{1 / 2}} .
$$

This represents a systematic bending which is just big enough to compete with random diffusion near the central parts of the contact volume. Near the edges of the reconnection zone it will be much more conspicuous. Also by our previous reasoning, we can expect that

$$
f_{r} \sim\left(\frac{v_{l}}{V_{A}}\right)^{2}\left(\frac{L_{x}}{l}\right)^{1 / 2}<1
$$

The field lines will bend drastically, with a large concentration of current and Ohmic dissipation, only in the reconnection zone itself. If we assume that the reconnection zone and current sheet are identical then

$$
V_{\text {rec }, \text { local }}=\frac{\eta}{\Delta}
$$

This is probably not quite right, but the effect of tearing modes appears to be only a modest enhancement of reconnection rates, judging from the results of Appendix $\mathrm{C}$, and the turbulent motions of reconnected field lines seem likely to disrupt modes with $k_{\|} \ll k_{\perp}$. We should nevertheless bear in mind that equation (34) may lead to an underestimate of the reconnection zone width, and an overestimate of Ohmic heating within it.

Since global reconnection speeds are given by equation (9), and since the accumulation of reconnected flux elements in the reconnection zone does not limit the pace of reconnection, we can use

$$
V_{\text {rec,global }} \approx k_{\|} L_{x} V_{\text {rec,local }} \approx k_{\perp} k_{\|} L_{x} \eta,
$$

where, as before, we have written the width of the reconnection zone in terms of the equivalent wavenumber in the turbulent cascade outside the reconnection zone. Using equation (回) we can rewrite this expression and equate it to the global reconnection speed given in equation (9). We find

$$
\Delta \approx l\left(\frac{\eta}{V_{A} l}\right)^{3 / 5}\left(\frac{V_{A}}{v_{l}}\right)^{2 / 5}\left(\frac{L_{x}}{l}\right)^{3 / 10}
$$

where we have assumed that $L_{x} \leq l$, for simplicity, and because this seems like the most likely case. The Ohmic dissipation rate, $\eta / \Delta^{2}$, is actually less than $k_{\|} V_{A}$ because the pace of reconnection is set by the escape of matter from the contact volume, rather than the reconnection zone. Consequently the ejection of matter from the reconnection zone is inhibited and the corresponding ejection velocity is less than $V_{A}$. In other words, the 
build-up of excess matter throughout the contact volume softens the pressure gradient so that escaping fluid reaches an ejection velocity $\sim V_{A}$ only after it has already left the reconnection zone.

One of the more striking aspects of our result of the global reconnection speed is that it is relatively insensitive to the actual physics of reconnection. Equation (9) only depends on the nature of the turbulent cascade. Although we have reached this conclusion by invoking a particular model for the strong turbulent cascade, we can see that any sensible model will give qualitatively similar results.

The conclusion that reconnection is fast, even when the local reconnection speed is slow, represents a triumph of global geometry over the slow pace of Ohmic diffusion. In the end, reconnection can be fast because if we consider any particular flux element inside the contact volume, assumed to be of order $L_{x}^{3}$, the fraction of the flux element that actually undergoes microscopic reconnection vanishes as the resistivity goes to zero.

\subsection{Reconnection for a modified spectrum of turbulence}

Our main conclusion, that reconnection is dramatically enhanced in the presence of a stochastic field, does not depend on the particular model of turbulence we adopt. Our work here assumes a locally generated turbulent spectrum which follows the scaling laws suggested by GS95. However, we depend only on the scaling law for power as a function of $k_{\perp}$ and the eddy anisotropy, that is $k_{\|}\left(k_{\perp}\right)$. The former is supported by observations of the solar wind. The latter depends on the assumption of locality of nonlinear interactions in wavenumber space and may be wrong (cf. Matthaeus et al. 1998). In Appendix D we discuss magnetic reconnection for an arbitrary power-law relation between $k_{\|}$and $k_{\perp}$ and

conclude that if $k_{\|} \sim k_{\perp}^{p}$ with $p>1 / 2$ then the reconnection rate is independent of the Lundquist number.

Consider now the processes that can modify the spectrum of turbulence. The turbulent spectrum may be changed due to: (a) a weak large scale magnetic field, (b) the injection of energy on small scales as a result of reconnection, (c) large scale discontinuities resulting from bringing magnetic fields with different directions into direct contact (by definition this is a necessary part of reconnection), (d) the backreaction of enhanced reconnection on the inverse cascade, and (e) the injection of field stochasticity from some other region.

We discussed (a) and (b) above. The first simply makes the field more chaotic and enhances reconnection rates, since the effective size of $l$ is reduced. The latter has a much weaker effect, since it has only a slight effect on the large scale field structure, but is likely to 
marginally increase reconnection speeds. Apropos (c), we expect that for strong magnetic fields brought into contact the efficiency of the coupling between eddies at different sides of the reconnection layer will be low. Consider, for example, two flux tubes at $\pi / 2$ angle. The eddies with $k_{\|, u p}$ in the upper flux tube will be exciting perturbations of the order $k_{\|, u p}^{-1}$ perpendicular to the magnetic field lines in the lower flux tube. However the time scales for perturbations with $k_{\perp, \text { low }}=k_{\|, \text {up }}$ will be very different from the time scale of the eddies providing excitation. Due to this mismatch in the time scales the crosstalk between eddies on opposite sides of the reconnection zone will be suppressed.

The high speed of reconnection given by equation (9) naturally leads to a question of self-consistency (our point (d) above). Is it reasonable to take the turbulent cascade suggested in GS95 when field lines in adjacent eddies are capable of reconnecting? It turns out that in this context, our estimate for $V_{\text {rec,global }}$ is just fast enough to be interesting. We note that when considering the intersection of nearly parallel field lines in adjacent eddies the acceleration of plasma from the reconnection layer due to the pressure gradient is not $k_{\|} V_{A}^{2}$, but rather $\left(k_{\|}^{3} / k_{\perp}^{2}\right) V_{A}^{2}$, since only the energy of the component of the magnetic field which is not shared is available to drive the outflow. On the other hand, the characteristic length contraction of a given field line due to reconnection between adjacent eddies is only $k_{\|} / k_{\perp}^{2}$. This gives an effective ejection rate of $k_{\|} V_{A}$. Since the width of the diffusion layer over a length $k_{\|}^{-1}$ is just $k_{\perp}^{-1}$, we can replace equation (9) with

$$
V_{\text {rec, }, \text { lobal }} \approx V_{A} \frac{k_{\|}}{k_{\perp}} .
$$

The associated reconnection rate is just

$$
\tau_{\text {reconnect }}^{-1} \sim V_{A} k_{\|},
$$

which in GS95 is just the nonlinear cascade rate on the scale $k_{\|}^{-1}$. However, this result is general and does not involve assuming that GS95 is correct. We will see in $\S 5.1$ that most of the energy liberated in reconnection goes into motions on length scales comparable to the dimensions of the reconnecting eddies, so this energy release will not short circuit the energy cascade described in GS95. On the other hand, we can invert this argument to see that reconnection can play an important role in preventing the buildup of unresolved knots in the magnetic field. Such structures could play a major role in inhibiting the cascade of energy to smaller scales, flattening the energy spectrum relative to the predictions of GS95. Our conclusion is that such structures will disappear as fast as they appear, supporting the notion that they play a limited role in the dynamics of MHD turbulence.

Finally, we note that if the magnetic field structure is driven by turbulence in another location, as when the footpoints of magnetic arcades are stirred by turbulent motions, then 
we can evaluate its effects in terms of the amplitude of field stochasticity and the scaling of structure anisotropy with scale. In the absence of any particular model for this process, we note that the robust nature of our conclusions, as shown in Appendix D, implies that under these circumstances reconnection will be sensitive to the amplitude of the induced field stochasticity, but not the details of the turbulent mixing process.

\section{Discussion}

\subsection{Energy dissipation}

The usual assumption for energy dissipation in reconnection is that some large fraction of the energy given up by the magnetic field, in this case $\sim \rho V_{A}^{2} L_{x}^{3}$, goes into heating the electrons. This is not the case here. Only a fraction, $\sim 1 /\left(k_{\|} L_{x}\right)$ of any flux element is

annihilated by Ohmic heating within the reconnection zone. Over the entire course of the reconnection event the efficiency for electron heating is no greater than

$$
\epsilon_{e} \lesssim \frac{\eta / \Delta}{V_{\text {rec,global }}}=\frac{V_{\text {rec }, \text { local }}}{V_{\text {rec,global }}}=\frac{1}{k_{\|} L_{x}} .
$$

or, from equations (9), (1), and (36),

$$
\epsilon_{e} \lesssim\left(\frac{V_{A} l}{\eta}\right)^{-2 / 5}\left(\frac{v_{l}}{v_{A}}\right)^{8 / 5}\left(\frac{l}{L_{x}}\right)^{4 / 5} .
$$

The electron heating within the current sheet will not be uniform, due to the presence of turbulence, the intermittent presence of reconnected flux, and any collective effects we have neglected here. To the extent that these are important they will also lower the electron heating efficiency by broadening the reconnection layer.

Naturally, the low value of $\epsilon_{e}$ is of little interest when ion and electron temperatures are tightly coupled. When this is not the case our model for reconnection may have testable consequences. As an example, we may consider advective accretion flows (ADAFs), following the general description given in Narayan and Yi (1995) in which advective flows can be geometrically thick, and optically thin with a small fraction of the dissipation going into electron heating. If, as expected, the magnetic pressure is comparable to the gas pressure in these systems, then a large fraction of the orbital energy dissipation occurs through reconnection events. If a large fraction of this energy goes into electron heating (cf. Bisnovatyi-Kogan \& Lovelace 1997) then the observational arguments in favor of ADAFs are largely invalidated. Our results suggest that reconnection, by itself, will not result in 
channeling more than a small fraction of the energy into electron heating 10

We can also estimate the efficiency of the conversion of magnetic field energy to high frequency Alfvén waves. One channel for the creation of such waves will be as waves radiated from the turbulence in the reconnection zone. The rate of energy emission is hard to estimate since the nature of the turbulence in the reconnection zone is so uncertain. Plausibly the energy lost from the this zone in the form of radiated Alfvén waves will be at least comparable to the Ohmic dissipation, but it is difficult to make a more precise estimate. On the other hand, the region immediately outside the reconnection zone will also be affected, via the constant interchange of reconnected flux and the subsequent relaxation of field lines just outside the reconnection zone. The total energy involved at any one time will be $\sim \rho V_{A}^{2} L_{x}^{2} \Delta$ and the rate of excitation of Alfvén modes should be of order $k_{\|} V_{\text {eject }}=k_{\|} V_{\text {rec,global }}$. This process will operate for a time $\sim L_{x} / V_{\text {rec,global }}$, so that the efficiency of wave energy generation will be

$$
\epsilon_{A} \sim \Delta k_{\|} \sim\left(\frac{\eta}{V_{A} l}\right)^{1 / 5}\left(\frac{V_{A}}{v_{l}}\right)^{2}\left(\frac{L_{x}}{l}\right)^{1 / 10} .
$$

The associated wave frequencies will be distributed between $k_{\|} V_{\text {rec,global }}$ and $V_{A} / \Delta$ depending on the nature of the turbulent zone, that is

$$
\frac{V_{A}}{l}\left(\frac{V_{A} l}{\eta}\right)^{2 / 5}\left(\frac{v_{l}}{V_{A}}\right)^{2 / 5}\left(\frac{l}{L_{x}}\right)^{1 / 5}<\omega_{A}<\frac{V_{A}}{l}\left(\frac{V_{A} l}{\eta}\right)^{3 / 5}\left(\frac{v_{l}}{V_{A}}\right)^{2 / 5}\left(\frac{l}{L_{x}}\right)^{3 / 10} .
$$

These limits differ by a factor of $\left(V_{A} l / \eta\right)^{1 / 5}$, which is large enough to make precise predictions difficult, but small enough that we have a qualitative sense of the results.

These waves are unlikely to affect of the topology of the magnetic field, since their typical scale is, at most, only $\lambda_{\|} \boxplus$. However, they may play a role in the anisotropic heating of ions in stellar coronae. In this connection we note recent observations of anisotropic velocity distributions for HII and OVI ions in the solar corona (Kohl et al. 1998) which they suggest may be due to the presence of high frequency (tens of kilohertz) Alfvén waves (see Cranmer, Field, \& Kohl 1998 for a detailed analysis).

\footnotetext{
${ }^{10}$ Other authors (e.g. Gruzinov 1998) have suggested that MHD turbulence in a collisionless, highly magnetized environment may not terminate on small scales in ion heating, so that ADAFs will necessarily deposit a large fraction of their heat into electrons. However, Gruzinov's calculation rests on treating the turbulence as a collection of independent linear waves, which is an unconvincing model for disturbances with a coherence time comparable to a single wave period.

${ }^{11}$ However, the large scale relaxation of magnetic field lines is likely to feed energy back into the turbulent cascade, providing a nonlinear feedback mechanism which will accelerate reconnection when the initial field stochasticity is small.
} 


\subsection{Dynamos}

To enable sustainable dynamo action and, for example, generate a galactic magnetic field, it is necessary to reconnect and rearrange magnetic flux on a scale similar to a galactic disc thickness within roughly a galactic turnover time $\left(\sim 10^{8}\right.$ years $)$. This implies that reconnection must occur at a substantial fraction of the Alfvén velocity. The preceding arguments indicate that such reconnection velocities should be attainable if we allow for a realistic magnetic field structure, one that includes both random and regular fields.

One of the arguments against traditional mean-field dynamo theory is that the rapid generation of small scale magnetic fields suppresses further dynamo action (e.g. Kulsrud \& Anderson 1992). Our results thus far show that a random magnetic field enhances reconnection by enabling more efficient diffusion of matter from the reconnection layer. This suggests that the existence of small scale magnetic turbulence is a prerequisite for a successful large scale dynamo. In other words, we are arguing for the existence of a kind of negative feed-back. If the magnetic field is too smooth, reconnection speeds decrease and the field becomes more tangled. If the field is extremely chaotic, reconnection speeds increase, making the field smoother. We note that it is common knowledge that magnetic reconnection can sometimes be quick and sometimes be slow. For instance, the existence bundles of flux tubes of opposite polarity in the solar convection zone indicates that reconnection can be very slow. At the same time, solar flaring suggests very rapid reconnection rates.

There is a general belief that magnetic dynamos operate in stars, galaxies (see Parker 1979) and accretion disks (see Balbus \& Hawley 1998). In stars, and in many accretion disks, the plasma has a high $\beta$, that is the average plasma pressure is higher than the average magnetic pressure. In such situations the high diffusivity of magnetic field can be explained by concentrating flux in tubes (Vishniac 1995a,b). This trick does not work in the disks of galaxies, where the magnetic field is mostly diffuse (compare Subramanian 1998) and ambipolar diffusion impedes the formation of flux tubes (Lazarian \& Vishniac 1996). This is the situation where our current treatment of magnetic reconnection is most relevant. However, our results suggest that magnetic reconnection proceeds regardless and that the concentration of magnetic flux in flux tubes via turbulent pumping is not a necessary requirement for successful dynamos stars and accretion discs.

We do not address here the controversial issue of the turbulent dynamo in clusters of galaxies. This was first suggested by (Jaffe 1980) and was elaborated in great detail by Ruzmaikin, Sokoloff \& Shukurov (1989), who claimed an excellent match between observations and predictions based on the Kazantsev (1968) theory of the turbulent dynamo. However, Goldshmidt \& Rephaeli (1993) found a large $\left(\sim 10^{20}\right)$ numerical error 
in the value of Ohmic diffusivity used by Ruzmaikin, Sokoloff \& Shukurov (1989), which formally invalidated their result. However, if it were possible to use the effective diffusivity determined by the reconnection rate instead of Ohmic diffusivity, then the theory of turbulent dynamo can be revived for clusters of galaxies. We postpone the discussion of this important question until our next paper.

Our results show that in the presence of MHD turbulence magnetic reconnection is fast, and this in turn allows the possibility of 'fast' dynamos in astrophysics (see the discussion of the fast dynamo in Parker (1992)). As the fluid conductivity tends to zero the relevant parallel wavelength also goes to zero, allowing a change of magnetic field topology via reconnection at a finite rate.

\subsection{Turbulent diffusion, particle diffusion, and self-excited reconnection}

It may seem from our discussion that this paper revives the concept of turbulent diffusion. However, a closer comparison between the concept original concept of turbulent diffusivity and our suggestion shows that the two are very different. Within the turbulent diffusivity paradigm, magnetic fields of different polarity were believed to filament and intermix on very small scales while reconnection was believed to proceed slowly. On the contrary, we have shown that the global speed of reconnection is fast if a moderate degree of magnetic field line wandering is allowed. The latter, unlike the former, corresponds to a realistic picture of MHD turbulence and does not entail prohibitively high magnetic field energies at small scales.

On the other hand, the diffusion of particles through a magnetized plasma is greatly enhanced when the field is mildly stochastic. There is an analogy between the reconnection problem and the diffusion of cosmic rays (Barghouty \& Jokipii 1996). In both cases charged particles follow magnetic field lines and in both cases the wandering of the magnetic field lines leads to efficient diffusion.

Finally, we note that observations of Solar flaring seem to show that such events start from some limited volume and spread as though a chain reaction from the initial reconnection region initiated a dramatic change in the magnetic field properties. Indeed, Solar flaring happens as if the resistivity of plasma were increasing dramatically as plasma turbulence grows (see Dere 1996 and references therein). In our picture this is a consequence of the increased stochasticity of the field lines rather than any change in the local resistivity. The change in magnetic field topology that follows localized reconnection provides the energy necessary to feed a turbulent cascade in neighboring regions. This kind of nonlinear 
feedback is also seen in the geomagnetic tail, where it has prompted the suggestion that reconnection is mediated by some kind of nonlinear instability built around modes with very small $k_{\|}$(cf. Chang 1998 and references therein). The most detailed exploration of nonlinear feedback can be found in the work of Matthaeus and Lamkin (1986), who showed that instabilities in narrow current sheets can sustain broadband turbulence in two dimensional simulations. Although our model is quite different, and relies on the three dimensional wandering of field lines to sustain fast reconnection, we note that the concept of a self-excited disturbance does carry over and may describe the evolution of reconnection between volumes with initially smooth magnetic fields.

\section{Conclusions}

In this paper we have explored the consequences of a small stochastic component for the reconnection of large scale magnetic fields. We have used a particular model of MHD turbulence, due to Goldreich and Sridhar (1995), in order to draw firm conclusions, but the main features of our results should follow from any model which is consistent with our observations of MHD turbulence in astrophysical systems. We have implicitly assumed that viscosity is less important than resistivity in setting the limits for any turbulent cascade. Although this should usually be the case for astrophysical plasmas, it is not guaranteed, and the effects of viscosity will need to be considered in more detail (Lazarian \& Vishniac 1999). Finally, we have assumed that we are dealing with a strong magnetic field, where motions that tend to mix field lines of different orientations are largely suppressed. The galactic magnetic field is usually taken to have grown via dynamo action from some extremely weak seed field (cf. Zel'dovich, Ruzmaikin \& Sokoloff 1983, Lazarian 1992, Kulsrud, Cen, Ostriker, \& Ryu 1997 and references contained therein). When the field is weak it can be moved as a passive scalar and its spectrum will mimic that of Kolmogorov turbulence. The difference between $\lambda_{\perp}$ and $\lambda_{\|}$vanishes, the field becomes tangled on small scales, and $V_{\text {rec,local }}$ becomes of the order of $V_{A}$. Of course, in this stage of evolution $V_{A}$ may be very small. However on the small scales $V_{A}$ will grow to equipartition with turbulent velocities on the turnover time of the small eddies. The enhancement of reconnection as $V_{A}$ increases accelerates the inverse cascade as small magnetic loops merge to form larger ones.

We list the principal results of this paper below.

First, the rate of magnetic reconnection is increased dramatically in the presence of a stochastic component to the magnetic field. This component arises naturally whenever turbulence is present. Even when the turbulent cascade is weak the resulting reconnection speed is independent of the Ohmic resistivity. 
Second, the argument that the rapid rise of random magnetic field associated with dynamo action results in the suppression of dynamo (Kulsrud \& Anderson 1992) is untenable since the increase of the random component of the magnetic field increases the reconnection rate. We conclude that dynamo is a self-regulating process.

Third, the second parameter in determining the reconnection speed is not some aspect of the microphysics, but the level of field stochasticity (or the large scale kinetic energy feeding the turbulent cascade). As reconnection proceeds the local turbulent cascade will grow stronger and the initial level of stochasticity will matter less and less. On the contrary, microphysical processes widely believed to speed up reconnection, i.e. anomalous resistivity, fail in interstellar conditions.

Fourth, since the speed of reconnection depends on the presence of small scale turbulence, non-resistive processes which terminate the turbulent cascade may slow reconnection down. As an example, we can cite neutral-ion collisions in a dense, partially ionized gas. On the other hand, the rate of collisions in a fully ionized plasma is irrelevant. Under typical astrophysical conditions $\nu \ll \eta$ for fully ionized plasmas. When collisions become very rare, the only effect is to eliminate the cascade of compressive modes, which are not important for reconnection.

Fifth, reconnection in a turbulent medium deposits only a small fraction of the magnetic field energy directly into heating electrons. Whether or not this is important depends on the rate of energy exchange between ions and electrons, and the unknown degree to which the turbulent energy cascade can avoid depositing its energy into electrons.

Sixth, regardless of the other arguments advanced here, there is a minimal reconnection speed, $\sim V_{A} \mathcal{R}_{L}^{-3 / 16}$, where $\mathcal{R}_{L} \equiv\left(V_{A} l / \eta\right)$, which is much faster than the Sweet-Parker estimate, even though it is still unrealistically slow.

Finally, the current sheet associated with reconnection is not substantially broadened from the Sweet-Parker result, even when reconnection is fast.

We acknowledge some helpful suggestions on the presentation of this material by the referee, Eugene Parker. We are thankful to B. Draine for helpful discussions and to Phil Kronberg for a critical reading of the manuscript. Elucidating comments by Bill Matthaeus and George Field are acknowledged. This work was supported in part by NASA grants NAG5-2858, NAG5-7030 (AL), NAG5-2773 (ETV), and NSF grant AST-9318185 (ETV). The final stages of this work were supported for one of us (AL) by CITA Senior Research Fellowship. ETV is grateful for the hospitality of MIT and the CfA during the early stages of this work, and CITA during its conclusion. 


\section{A. The Power Spectrum of Magnetohydrodynamic Turbulence}

Here we discuss a model for MHD turbulence first proposed in GS95. Although their

work included an explicit calculation of coupling constants based on treating the turbulent motions as shear Alfvén waves, the important parts of the model depend only on some rather general scaling arguments, which we will repeat here. These arguments depend on assuming that power is transferred locally in Fourier space, which is a convenient, but not rigorously justified, assumption.

We begin by noting that the first simple model of incompressible MHD turbulence was proposed independently by Iroshnikov (1963) and Kraichnan (1965) based on the interactions of triads of waves (see also Diamond and Craddock 1990). In this picture the energy transfer rate is roughly

$$
\tau_{n l}^{-1} \approx \frac{\left(k v_{k}\right)^{2}}{\omega_{A}}
$$

where $k$ is the magnitude of a wavevector, $v_{k}$ is the rms fluid velocity contributed from power on the scale $k^{-1}, \omega_{A} \equiv k_{\|} V_{A}$ is the Alfvén wave frequency, $k_{\|}$is the wavevector component parallel to the magnetic field direction, and $V_{A}$ is the Alfvén velocity. In the original picture the power was assumed to spread isotropically in wavevector space. As long as $v_{k}<V_{A}$ this expression for the nonlinear time scale will be less than $k v_{k}$, that is, the magnetic field reduces the cascade of energy to higher wavenumbers.

In a recent series of papers Goldreich and Sridhar (Sridhar and Goldreich 1994; Goldreich and Sridhar 1995; Goldreich and Sridhar 1997) questioned the validity of this picture and argued that the diffusion of power towards larger values of $k_{\|}$is strongly suppressed. Their original claim that three wave interactions are completely suppressed has been strongly criticized (Montgomery and Matthaeus 1995, Ng \& Bhattacharjee 1996). However, in their most recent paper (Goldreich and Sridhar 1997) they showed convincingly that the effect of residual three wave couplings is consistent with a picture in which the basic nonlinear time scale is set by equation (A1) but with an anisotropic spectrum in which virtually all of the transfer of power between modes moves energy towards larger $k_{\perp}$ while leaving $k_{\|}$unchanged. They proposed calling this regime 'intermediate turbulence', since while the nonlinear decay rate is identical to the usual expression for weak turbulence among dispersive waves, in this case the higher order mode couplings are all comparably important. If we invoke the constancy of the local energy flux through the cascade, $v_{k}^{2} / \tau_{n l}$, as a function of scale, then from equation (A1) we see that in this regime

$$
v_{k} \propto k_{\perp}^{-1 / 2}
$$

where we have assumed that $k_{\perp}>>k_{\|}$. 
As the power cascades to larger values of $k_{\perp}$ the magnetic field becomes progressively less important in the mode dynamics. Eventually we have

$$
k_{\|} V_{A} \leq k v_{k} .
$$

In this limit the motions are no longer wave-like, the magnetic field exerts only a weak influence on the dynamics, and the fluid motions resemble ordinary hydrodynamical turbulence with a nonlinear time scale $\sim k v_{k}$. Since $k_{\|}$is no longer privileged, the cascade of power is in the direction of increasing isotropy. Since $k_{\perp} \gg k_{\|}$this implies an increase in $k_{\| \cdot}$

If energy is injected on some scale $l$, with $v_{l} \leq V_{A}$, then we expect the cascade to transfer energy to larger $k_{\perp}$ until the condition expressed in equation (A3) is satisfied. At this point the turbulence is no longer wavelike (since $\tau_{n l}^{-1} \sim k_{\|} V_{A}$ ). However, since turbulence tends towards isotropy when the magnetic field is completely negligible, we expect $k_{\|}$and $k_{\perp}$ to increase in tandem so that equation (A3) is just marginally satisfied. This is the regime of strong turbulence described in GS95. At all smaller parallel wavelengths fluid motions bend magnetic field lines easily. Consequently we expect most of the power in energy spectrum to be centered around wavenumbers such that

$$
k_{\|} \sim k_{\perp} \frac{v_{k}}{V_{A}}
$$

We approximate the energy transfer rate for the turbulent cascade, $\dot{\mathcal{E}}$, for $v_{l} \leq V_{A}$ as

$$
\dot{\mathcal{E}} \approx \frac{v_{l}^{4}}{l V_{A}}
$$

if $v_{l} \leq V_{A}$ on the scale $l$. The usual hydrodynamic choice $\dot{\mathcal{E}} \approx \frac{v_{l}^{3}}{l}$ is valid otherwise, although not relevant for our present discussion. When the magnetic field is weak and the largest scales in the turbulent cascade are essentially hydrodynamic then we can identify $l$ with the scale of equipartition so that $v_{l}^{2} \sim V_{A}^{2}$.

Conservation of energy in the turbulent cascade implies that $\dot{\mathcal{E}}=v_{k}^{2} / \tau_{n l}=$ const and is only determined by large scale motions. If $l$ is the large scale eddy size, and $v_{l}<V_{A}$ then we use equation (A5) to find $\dot{\mathcal{E}}$. From equation (A1) we find that

$$
\dot{\mathcal{E}}=\frac{v_{l}^{4}}{l V_{A}} \sim \frac{v_{k}^{2}}{\tau_{n l}} \sim v_{k}^{4} \frac{k_{\perp}^{2}}{k_{\|} V_{A}}
$$

Combining equations ( $\mathrm{A} 4)$ and (A6) we obtain

$$
k_{\|} \approx l^{-1}\left(k_{\perp} l\right)^{2 / 3}\left(\frac{v_{l}}{V_{A}}\right)^{4 / 3} .
$$


Substituting this in equation (A1) gives the energy transfer rate

$$
\tau_{n l}^{-1} \approx \frac{v_{l}}{l}\left(k_{\perp} l\right)^{2 / 3}\left(\frac{v_{l}}{V_{A}}\right)^{1 / 3},
$$

while the rms fluid velocity on the scale $k$ follows from equation (A4)

$$
v_{k} \approx v_{l}\left(k_{\perp} l\right)^{-1 / 3}\left(\frac{v_{l}}{V_{A}}\right)^{1 / 3}
$$

Had we assumed an isotropic distribution of power in $\vec{k}$ space (that is, had we failed to distinguish between $k_{\perp}$ and $k_{\|}$) we would have recovered the Kraichnan spectrum, with $v_{k} \propto k^{-1 / 4}$. Instead, we find $v_{k} \propto k_{\|}^{-1 / 2}$. Curiously enough, this anisotropic cascade looks qualitatively like the Kolmogorov spectrum when expressed in terms of $k_{\perp}$. This becomes less surprising if one recalls that the magnetic field does not influence motions that do not bend it. We also note that for the characteristic $k_{\|}$given in equation (A7) the interaction between different modes can be viewed, with equal validity, as a wave-wave interaction, an eddy-eddy interaction, or a wave-eddy interaction. That the first two give roughly equal nonlinear time scales is immediately apparent from equations (A1), and (A4). That the third point of view is also equivalent follows from the work of Similon and Sudan (1989) who showed that the dissipation of Alfvén waves in a stochastic field can be estimated by replacing the microscopic Ohmic resistivity by the diffusion coefficient for waves traveling along the field lines. In terms of the turbulent cascade envisioned here, this is equivalent to making the substitution

$$
\eta \rightarrow \eta_{t u r b} \approx \Delta^{2} \omega_{A}
$$

where $\Delta$ is the typical scale of perpendicular displacements of the field lines, and also the correlation length for such displacements. In our model, for typical modes, $k_{\perp} \Delta \sim 1$. It follows that $k_{\perp}^{2} \eta_{\text {turb }}$ is equal to other estimates of the nonlinear interaction time scale.

The maximum $k_{\perp}$ can be obtained from the condition that the energy transfer rate (see eq. (A8)) be greater than the resistive dissipation rate $k_{\perp}^{2} \eta$. We get

$$
k_{\perp} l<k_{\perp, c} l \approx \frac{v_{l}}{V_{A}}\left(\frac{V_{A} l}{\eta}\right)^{3 / 4} .
$$

Analogously, viscosity will be negligible as long as $\tau_{n l}^{-1}$ is greater than the viscous dissipation rate $k_{\perp}^{2} \nu_{\perp}$, which implies that

$$
k_{\perp} l<\frac{v_{l}}{V_{A}}\left(\frac{V_{A} l}{\nu_{\perp}}\right)^{3 / 4}
$$

where we have written the viscous diffusivity as $\nu_{\perp}$ to emphasize that it is the viscosity perpendicular to magnetic field lines which enters into this estimate. For a pure plasma the 
viscous diffusivity may be large when the density and magnetic field are both low, but it will decrease as the field strength increases.

In partially ionized gas, collisions with neutrals provide an additional source of viscosity. In this case the ambipolar diffusion coefficient $\eta_{a m b}$ should be used instead of $\eta$ in Eq. (A11). For typical conditions in the cold interstellar medium (see Vishniac \& Lazarian 1998)

$$
\eta_{a m b} \approx 5.3 \times 10^{21} n_{0.03}^{-1} N_{30}^{-1} T_{100}^{-1 / 2} B_{5}^{2} \mathrm{~cm}^{2} / \mathrm{s}
$$

where the letters with subscripts denote the normalized values of the parameters adopted. In other words, the electron density $n$ is normalized on the density $0.03 \mathrm{~cm}^{-3}$, neutral density $N$ is normalized by $30 \mathrm{~cm}^{-3}$, typical temperature $T$ is assumed $100 \mathrm{~K}$, while typical magnetic field $5 \times 10^{-6}$ Gauss. Such a high diffusivity implies $k_{\max } l$ is only $10^{3}$ for $l=30 \mathrm{pc}$, so that turbulence will be damped at $\sim 0.03$ pc scales in the cold interstellar medium.

Rather high values of diffusivity follow from collisionless damping (see Foote \& Kulsrud 1969), but this only affects compressional modes in the cascade. GS95 showed that very little energy is transferred to the compressional modes from the shear modes on scales for which $k_{\perp} \gg k_{\|}$. Consequently, the suppression of compressional modes has little direct impact on the small scale structure of the magnetic field.

The minimum $k_{\perp}$ covered by these scaling laws follows from equation (A7). We find that

$$
k_{\perp} l>\left(\frac{V_{A}}{v_{l}}\right)^{2} .
$$

Larger perpendicular scales will be described by weak Alfvenic turbulence.

Are these scaling laws consistent with observations? The Sun provides our best example of strong MHD turbulence, but current observations of the solar corona and photosphere do not significantly constrain the power spectrum of the inertial range. On the other hand, the solar wind contains dynamical disturbances on a broad range of scales. Barnes (1979) has advocated that these should be interpreted as Alfvén waves emitted from the solar corona and undergoing only weak dissipation in their outward journey. However Coleman (1968) has argued that the shorter wavelength disturbances are most naturally interpreted as a turbulent spectrum, and the solar wind shows evidence for local dissipation on small scales. A recent analysis by Leamon et al. (1998) supports the latter interpretation and argues that between $1.6 \times 10^{-4}$ and $4 \times 10^{-2} \mathrm{~Hz}$ perturbations in the solar wind are in the inertial range of strong MHD turbulence. (Quoted frequencies are measured at a spacecraft essentially stationary in a moving solar wind.) The power spectrum in this range has an index of roughly -0.7 , consistent with a Kolmogorov index of $-2 / 3$. Since the solar wind advects disturbances over the spacecraft radially, while the unperturbed magnetic 
field vector is strongly tilted by the Sun's rotation, this is essentially a measurement of the scaling law with respect to $k_{\perp}$. The observations also indicate that the turbulence is strongly anisotropic. This is in qualitative agreement with GS95, although a quantitative comparison would require an estimate of the fraction of the energy contained in nonlinear magnetosonic waves, and the local value of $P_{\text {mag }} / P_{\text {gas }}$. This raises questions that have little impact on the nature of the small scale stochastic magnetic field structure, and we will not pursue them further.

MHD turbulence in the ISM can be studied via scintillations. These measurements (see Spangler 1998) reveal that the density irregularities responsible for radio wave scintillation are elongated and aligned along the magnetic field. The scintillations themselves are believed to result from entropy fluctuations that arise from the mixing of plasma elements having different specific entropies. The entropy acts as a passive scalar and consequently its spectrum assumes the form of the turbulent energy spectrum (see Lesieur 1990). As the sharpest entropy gradients lie in the plane perpendicular to the magnetic field, we would expect to see the spectrum $k^{-5 / 3}$. It is encouraging that the measurements (see Armstrong, Rickett \& Spangler 1997) do get such a spectrum. Moreover, the elongation of fluctuations along the field lines follows from the disparity between $k_{\|}$and $k_{\perp}$. This may also serve as a hint in favor of Goldreich and Sridhar's model[2].

Numerical simulations also seems to be consistent with the GS95 model. The high wavenumber resistive cutoff is given by Eq. (A11). Together with Eq. (A7) this sets the upper limit to $k_{\|}$:

$$
k_{\|, c} \approx l^{-1}\left(\frac{v_{l}}{V_{A}}\right)^{2}\left(\frac{l V_{A}}{\eta}\right)^{1 / 2} .
$$

We immediately see that, if $b$ is the perturbation of magnetic field at the scale $l$,

$$
\frac{k_{\|, c}}{k_{\perp, c}} \approx \frac{v_{l}}{V_{A}}\left(\frac{\eta}{l V_{A}}\right)^{1 / 4} \approx \frac{b}{B}\left(\frac{\eta}{l V_{A}}\right)^{1 / 4},
$$

which is similar to the scaling relation ${ }^{\mathbb{3}}$ reported in Matthaeus et al (1998).

\footnotetext{
${ }^{12}$ The range of scales over which the spectrum has this form is hotly debated. The emissivity of HI over the scale 10-100 pc has a shallower spectrum in the velocity space defined by galactic rotation (Lazarian 1995). Lazarian \& Pogosyan (1999) claim that this spectrum is consistent with the Goldreich \& Sridhar's model of turbulence. However, here we are mostly interested in the smallest scales.

${ }^{13}$ Strictly speaking, in Matthaeus et al (1998) the relation $k_{\|, c} / k \propto b / B$, where $k=\left(k_{\|, c}^{2}+k_{\perp, c}^{2}\right)^{1 / 2}$, was reported. The scaling above differs from this by $\sim(b / B)^{1 / 4}$, which for the limited dynamical range available provides a difference of the order $30 \%$. However, the difference may not be statistically significant. Peter Goldreich (private communication) attracted our attention to the fact that in Matthaeus et al (1998) $k_{\|}$
} 


\section{B. Previously explored ways to speed up reconnection}

\section{B.1. Anomalous resistivity}

Anomalous resistivity is present in the reconnection layer when the field gradient is so sharp that the electron drift velocity is of the order of thermal velocity of ions $u=(k T / m)^{1 / 2}$. For a detailed discussion of the physics see Parker (1979). The condition for appearance of anomalous resistivity is

$$
j>j_{c r}=N e u
$$

If the current sheet has a width $\delta$ with a change in the magnetic field $\Delta B$ then

$$
4 \pi j=\frac{c \Delta B}{\delta}
$$

The effective resistivity increases nonlinearly as $j$ becomes greater than $j_{c r}$, thereby broadening the current sheet. We can therefore assume that $j$ stays of the order of $j_{c r}$, that is

$$
\delta \approx \frac{c \Delta B}{4 \pi N e u}
$$

Expressing $\delta$ through ion cyclotron radius $r_{c}=(m u c) /\left(e B_{t o t}\right)$, where $B_{t o t}$ is the total magnetic field (including any shared component) one gets

$$
\delta \approx r_{c}\left(\frac{V_{A}}{u}\right)^{2} \frac{\Delta B}{B_{t o t}}
$$

which agrees with Parker (1979) up to the factor $\Delta B / B_{\text {tot }}$, which is equal 1 in Parker's treatment.

We can derive a reconnection speed by combining mass conservation and equation (B4). We get

$$
V_{\text {rec }} \approx V_{A} \frac{r_{c}}{L_{x}}\left(\frac{V_{A}}{u}\right)^{2} \frac{\Delta B}{B_{t o t}}
$$

Equation (B5) shows that the enhanced reconnection velocity is much less than the Alfven velocity if the characteristic scale of the problem $L_{x}$ is much greater than the ion Larmor (cyclotron) radius. In general, "anomalous reconnection" is important when the

is defined solely in terms of the background field and therefore if $k_{\|}$is smaller than $k_{\perp} \theta$, where $\theta$ is the typical large scale bending angle, the local value of $k_{\|}$is not measured by this statistic. This will bias the measurements of the anisotropy towards a linear relationship. Definitely, new, more elaborate numerical tests are needed! 
thickness of the reconnection layer in the Sweet-Parker reconnection scheme is less than $\delta$. This means that when

$$
\delta>\left(\frac{L_{x} \eta}{V_{A}}\right)^{1 / 2},
$$

anomalous effects are important. The condition (B6) is easily fulfilled in the laboratory, where anomalous effects are widely observed. However, for typical interstellar magnetic fields the Larmor radius $r_{c}$ is $\sim 10^{7} \mathrm{~cm}$. For interstellar Ohmic diffusivities $\eta \approx 10^{9} \mathrm{~cm}^{2} / \mathrm{sec}$ and $V_{A} \approx u \sim 10^{6} \mathrm{~cm} / \mathrm{sec}$ we find that anomalous resistivity becomes important if

$$
L_{x}<\frac{\delta^{2} V_{A}}{\eta} \approx 10^{11} \mathrm{~cm} \approx 0.01 \mathrm{AU},
$$

which is a very small scale for the ISM. All in all, anomalous effects are unlikely to be important for interstellar reconnection if the Sweet-Parker scheme is involved.

In the presence of magnetic field turbulence the exact value of resistivity is much less significant. Nevertheless, anomalous resistivity may be important within the reconnection zone itself. By comparison with equation (36). we can see that anomalous effects will be important if

$$
r_{c}>l\left(\frac{\eta}{V_{A} l}\right)^{3 / 5}\left(\frac{V_{A}}{v_{l}}\right)^{2 / 5}\left(\frac{L_{x}}{l}\right)^{3 / 10},
$$

or assuming $V_{A} \sim v_{l}$ and $L_{x} \sim l$

$$
l<10^{13} \mathrm{~cm} \approx 1 \mathrm{AU}
$$

Unfortunately, under normal conditions this is still a very small length scale. If $l$ is less than $\sim 1 \mathrm{AU}$ the speed of reconnection can be found from the condition $V_{\text {rec,local }} \lambda_{\|} \approx V_{A} \delta$, where $\lambda_{\|} \sim k_{\|}^{-1}$ is given by equation (A7) and we assume $k_{\perp}$ that enters this formulae $\sim \delta^{-1}$. Substituting $\delta$ from equation (B4) we get

$$
V_{r e c, l o c a l} \approx V_{A}\left(\frac{r_{c}}{l}\right)^{1 / 3}\left(\frac{\Delta B}{B_{t o t}}\right)^{1 / 3}\left(\frac{V_{A}}{u}\right)^{2 / 3}\left(\frac{v_{l}}{V_{A}}\right)^{4 / 3}
$$

Similarly, in the presence of the ambipolar diffusion in the gas with low degree of ionization the thickness of the reconnection layer becomes just barely greater than the ion Larmor radius (Vishniac \& Lazarian 1998) so that we might expect the onset of anomalous effects, but even if they were marginally important they would not change the reconnection velocity significantly. In general we see that anomalous effects are of limited importance for reconnection in the interstellar medium.

Recently this topic was revisited by Biskamp et al. (1997) and Shay et al. (1998) who showed that in a collisionless plasma the minimum width for the ion flow is actually the ion 
skin depth $c / \omega_{p i}$ rather than the ion cyclotron radius. Since the former is just $V_{A} / u_{i}$ times the latter, this does not affect the conclusion that anomalous effects cannot explain fast reconnection in astrophysical plasmas t4t.

\section{B.2. Bohm diffusion}

Bohm diffusion is phrase which describes a process which is ubiquitous in laboratory plasmas, but which lacks a good theoretical explanation. Its characteristic feature is that ions appear to scatter about once per Larmor precession period. The resulting particle diffusion destroys the 'frozen-in' condition and allows significant larger magnetic field line diffusion. For an ion thermal velocity $u \approx(k T / m)^{1 / 2}$ and a cyclotron radius $r_{c}=m u c / q B$, where $q$ is the ion charge, the Bohm particle diffusion coefficient is $\eta_{\mathrm{B}} \approx u r_{c} \approx k T(c / q B)$. If we are concerned instead with the diffusion of field lines, we need to substitute $V_{A}^{2}$ for $k T / m$ so that $\eta_{\text {Bohm }} \sim V_{A} r_{c}$. Since $V_{A}$ is of order $u$ in the ISM this has no effect on our estimates. In the presence of Bohm diffusion, this coefficient should be used in place of Ohmic diffusivity (Parker 1979). However, we note that even if we make this substitution, it can produce fast reconnection, of order $V_{A}$, only if $r_{c} \sim L_{x}$. It therefore fails as an explanation for fast reconnection for the same reason that anomalous resistivity does. The difference between the two processes is that anomalous resistivity vanishes when the scales involved become larger than the threshold values given by equations (B7) and (B9), while we cannot define such a threshold for Bohm diffusion.

We may repeat all our calculations in the presence of Bohm diffusion by substituting

$\eta_{\text {Bohm }}$ instead of $\eta$ and as $\eta_{\text {Bohm }} \gg \eta$, the lower limit for the reconnection rates will increase by a substantial factor $\left(\nu_{\mathrm{Bohm}} / \eta\right)^{3 / 16} \gg 1$.

A major shortcoming of this idea is that it is not at all clear that Bohm diffusion is as omnipresent under astrophysical conditions as in laboratory plasmas. Moreover, since our present study suggests that the actual reconnection rate does not depend on the value of diffusivity, this substitution is probably irrelevant.

\footnotetext{
${ }^{14}$ Shay et al. also found that the reconnection speed in their simulations was independent of $L_{x}$, which would suggest that something like Petschek reconnection emerges in the collisionless regime. However, their dynamic range was small and the ion ejection velocity increased with $L_{x}$, with maximum speeds approaching $V_{A}$ for their largest values of $L_{x}$. Assuming that $V_{A}$ is an upper limit on ion ejection speeds we may expect a qualitative change in the scaling behavior of their simulations at slightly larger values of $L_{x}$.
} 


\section{Tearing modes}

Tearing modes are a robust instability connected to the appearance of narrow current sheets (Furth, Killeen, \& Rosenbluth 1963). The resulting turbulence will broaden the reconnection layer and enhance the reconnection speed. Here we give an estimate of this effect and show that while it represents a significant enhancement of Sweet-Parker reconnection of laminar fields, it changes our results by only a small amount. One difficulty with many earlier studies of reconnection in the presence the tearing modes stemmed from the idealized two dimensional geometry assumed for reconnection (Furth, Killeen, \& Rosenbluth 1963). In two dimensions tearing modes evolve via a stagnating non-linear stage related to the formation of magnetic islands. This leads to a turbulent reconnection zone (Matthaeus \& Lamkin 1985), but the current sheet remains narrow and its effects on the overall reconnection speed are unclear. This nonlinear stagnation stage does not emerge when realistic three dimensional configurations are considered. Indeed, it is easy to see that instead of islands one finds nonlinear Alfvén waves in three dimensional reconnection layers. Therefore the tearing instability proceeds at high rates determined by its linear growth while the resulting magnetic structures propagate out of the reconnection region at the Alfvén speed.

The dominant mode will be the longest wavelength mode, whose growth rate will be

$$
\gamma \approx \frac{\eta}{\Delta^{2}}\left(\frac{V_{A} \lambda_{\|}}{\eta}\right)^{2 / 5}
$$

The transverse spreading of the plasma in the reconnection layer will start to stabilize mode when its growth rate is comparable to the transverse shear $V_{A} / \lambda_{\|}$(Bulanov, Sakai, \& Syrovatskii 1979). At this point we have

$$
V_{r e c, l o c a l} \approx \gamma \Delta \approx V_{A} \frac{\Delta}{\lambda_{\|}}
$$

For a laminar magnetic field we have $L_{x}=\lambda_{\|}$and these two equations imply

$$
V_{\text {rec }}=V_{A}\left(\frac{\eta}{V_{A} L_{x}}\right)^{3 / 10}
$$

This is substantially faster than the usual Sweet-Parker formula, but still quite slow. We note in passing that the value of $\Delta / \lambda_{\|}$we assume here is below the minimum value proposed by Furth et al. (1963). However, it is consistent with the more accurate calculations of Van Hoven and Cross (1971).

\footnotetext{
${ }^{15}$ As a consequence of stabilization, tearing mode reconnection is stationary in a time-averaged sense.
} 
If we consider instead a stochastic field with $\lambda_{\|} \ll L_{x}$, then we can express the local reconnection rate in terms of $k_{\|} \sim \lambda_{\|}^{-1}$. Substituting $k_{\|}$from equation (A7) and assuming $k_{\perp} \sim \Delta^{-1}$ we get

$$
V_{\text {rec,local }} \approx V_{A}\left(\frac{v_{l}}{V_{A}}\right)^{3 / 4}\left(\frac{\eta}{V_{A} l}\right)^{3 / 16},
$$

while

$$
k_{\|} l \sim\left(\frac{v_{l}}{V_{A}}\right)^{5 / 2}\left(\frac{V_{A} l}{\eta}\right)^{3 / 8}
$$

and

$$
k_{\perp} l \sim\left(\frac{v_{l}}{V_{A}}\right)^{7 / 4}\left(\frac{V_{A} l}{\eta}\right)^{9 / 16} .
$$

The tearing instability broadens the reconnection region. For a completely ionized gas $k_{\perp}<k_{\perp, \max }$, and equation (C4) holds, provided that

$$
\frac{V_{A} l}{\eta}>\left(\frac{v_{l}}{V_{A}}\right)^{4},
$$

which is nearly always true. In a partially ionized gas the tearing modes will be partly suppressed, and this mechanism for increasing $V_{\text {rec,local }}$ will be relatively ineffective.

Equation (C4) is the speed of reconnection for each flux element in a fully ionized gas, using Goldreich and Sridhar's model for the MHD turbulent cascade, and assuming the Sweet-Parker topology for the reconnection sheet. Equations (13) and (C4) are not dramatically different. The exponent of the Lundquist number, $\mathcal{R}_{L} \equiv\left(V_{A} l / \eta\right)$ changes by only $1 / 16$. We also note that the frequency of reconnection, in this case the tearing mode growth rate, is $\sim V_{A} / \lambda_{\|}$, so once more we can treat the field line structure as essentially static.

\section{Turbulence with an arbitrary spectrum}

Although in the main body of the paper we presented arguments in favor of Goldreich \& Sridhar's (1995) model of turbulence, it is worthwhile considering reconnection when the relation between $k_{\|}$and $k_{\perp}$ is of the form

$$
k_{\|} \approx l^{-1}\left(k_{\perp} l\right)^{p}\left(\frac{v_{l}}{V_{A}}\right)^{m},
$$

where $p$ and $m$ are unspecified positive constants. For GS $p=2 / 3$, and $m=4 / 3$. On the other hand, Matthaeus et al. (1998) have criticized the model of Goldreich and Sridhar, 
partly on the basis of numerical simulations, and proposed an alternative model in which $p=m=1$ 开. Using equation (D1) instead of eq. (5), we get

$$
\left\langle y^{2}\right\rangle^{1 / 2}=\frac{(2 x / p)^{1 / p}}{l^{(1-p) / p}}\left(\frac{v_{l}}{V_{A}}\right)^{m / p},
$$

which implies an outflow layer width of

$$
\left\langle y^{2}\right\rangle^{1 / 2} \sim l\left(\frac{L_{x}}{l}\right)^{1 / p}\left(\frac{v_{l}}{V_{A}}\right)^{m / p}
$$

when $l>L_{x}$ and

$$
\left\langle y^{2}\right\rangle^{1 / 2} \sim\left(L_{x} l\right)^{1 / 2}\left(\frac{v_{l}}{V_{A}}\right)^{m / p}
$$

when $L_{x}>l$ (compare to (77) and (8)). Therefore the upper limit on $V_{\text {rec }}$ imposed by large scale field line diffusion is given not by (9) but

$$
V_{r e c}<V_{A} \min \left[\left(\frac{L_{x}}{l}\right)^{1 / p-1},\left(\frac{l}{L_{x}}\right)^{1 / 2}\right]\left(\frac{v_{l}}{V_{A}}\right)^{m / p}
$$

which shows that for a generous range of $p$ and $m$ the outflow of fluid from the reconnection zone does significantly limit reconnection speeds.

In order to recover limits on $p$ or $m$ which are consistent with fast reconnection we need to consider effects which might provide more stringent constraints on the reconnection speed. Some of our discussion cannot be reproduced for the general case without a model for the transfer of energy, as well as a scaling of eddy anisotropy. However, one important limit comes from considering the limits on small scale reconnection events. For the general case we replace equation (13) with

$$
V_{\text {rec }, \text { local }} \sim V_{A}\left(\frac{\eta}{V_{A} l}\right)^{\frac{1-p}{2-p}}\left(\frac{v_{l}}{V_{A}}\right)^{m /(2-p)} .
$$

Following the line of reasoning used in the main text requires that the probability a given magnetic field line will return back to the reconnection zone is small. Formally this means that $p<1$. However, for $p=1$ the local reconnection speed has already reached its maximal value $V_{A}$, since in the case of locally isotropic eddies the reconnection geometry is basically the one proposed by Petschek (1964). For larger values of $p$ reconnection proceeds at $V_{A}$.

\footnotetext{
${ }^{16}$ Note, however, that the scaling of eddy anisotropy seen in their simulations is in agreement with GS95, once allowance is made for the existence of weak turbulence on large scales (cf. the discussion at the end of Appendix A of this paper).
} 
Since $p>1$ corresponds to a spectrum in which eddies become increasingly elongated across magnetic field lines on small scales, this choice is almost certainly unphysical as well.

For $p<1$ the Ohmic diffusivity provides the following estimate for the reconnection rate (compare with equation (16)

$$
V_{\text {rec }, \text { global }}=k_{\|} L_{x} V_{\text {rec }, \text { local }}=V_{A}\left(\frac{V_{A} l}{\eta}\right)^{(2 p-1) /(2-p)} \frac{L_{x}}{l}\left(\frac{v_{l}}{V_{A}}\right)^{3 m /(2-p)},
$$

which shows that the reconnection velocity does not depend on Lundquist number provided that $p>1 / 2$. Otherwise Ohmic diffusion will limit the reconnection rate to the value given by eq. (D7). This will reach the Sweet-Parker reconnection rate when $p=0$, that is, when the parallel wavelength does not increase as we go down the turbulent cascade. Physically, this is equivalent to assuming that the magnetic field lines are infinitely stiff, even when the local kinetic energy density is comparable to the magnetic field energy density.

We note that even taking the relatively modest value of $p=1 / 2$ implies that the magnetic field suppresses bending motions far more efficiently than one would suppose from simple energetic arguments.

Finally, it is useful to see how these arguments would change if the Kraichnan spectrum turns out to be correct. We have earlier explained why we do not expect this to be the case, but this is the default model for many researchers. Also, it turns out that this model cannot be found as a special case of equation (D1). Instead, while most of the power is contained in modes with $k_{\perp} \sim k_{\|}$, the amplitude of the waves is given by

$$
\frac{v_{k}}{\omega_{A}} \approx k^{-1} \frac{v_{k}}{V_{A}} \ll k^{-1}
$$

which sets this case apart from the strongly nonlinear cascade, where the wave amplitude is $\sim k_{\perp}^{-1}$. If we combine the assumption of isotropy in wavevector space with equations (A1) and (A5) we find the usual result, for the Kraichnan spectrum, that

$$
v_{k} \sim v_{l}(k l)^{-1 / 4}
$$

Combining this with equation (D8) we see that the characteristic displacement on a scale $k^{-1}$ is

$$
<y^{2}>^{1 / 2} \sim \frac{v_{l}}{V_{A}} \frac{l}{(k l)^{5 / 4}} .
$$

This implies a field line diffusion, analogous to equation (5) of

$$
\frac{d\left\langle y^{2}\right\rangle}{d x} \sim l\left(\frac{\left\langle y^{2}\right\rangle}{l^{2}}\right)^{3 / 4}\left(\frac{v_{l}}{V_{A}}\right)^{2}
$$


which in turn leads to an outflow layer width, for $L_{x}<l$, of

$$
\left\langle y^{2}\right\rangle^{1 / 2} \sim l\left(\frac{L_{x}}{l}\right)^{2}\left(\frac{v_{l}}{V_{A}}\right)^{4} .
$$

The upper limit on $V_{\text {rec,global }}$ set by field line diffusion is therefore

$$
V_{\text {rec,global }}<V_{A} \frac{L_{z}}{l}\left(\frac{v_{l}}{V_{A}}\right)^{4}
$$

To see that this is the actual speed of reconnection we need to evaluate $V_{\text {rec,local }}$. The Kraichnan spectrum will be truncated due to resistivity at a wavenumber given by

$$
k_{c} \sim l^{-1}\left(\frac{v_{l}}{V_{A}}\right)^{4 / 3}\left(\frac{V_{A} l}{\eta}\right)^{2 / 3} .
$$

Using the usual Sweet-Parker formula for the local reconnection speed we get

$$
V_{\text {rec,local }} \sim V_{A}\left(\frac{v_{l}}{V_{A}}\right)^{2 / 3}\left(\frac{\eta}{V_{A} l}\right)^{1 / 6} .
$$

We can obtain the same estimate by ignoring the spectral cutoff, and calculating the appropriate value of $k$ by requiring that $\eta\left\langle y^{2}\right\rangle^{-1 / 2}$ equal the local Sweet-Parker reconnection speed. The upper limit on the global reconnection speed is recovered by multiplying $V_{\text {rec,local }}$ by $k L_{x}$. We obtain

$$
V_{\text {rec,global }}<V_{A}\left(\frac{V_{A} l}{\eta}\right)^{1 / 2} \frac{L_{x}}{l}\left(\frac{v_{l}}{V_{A}}\right)^{4} .
$$

Comparing equations (D13) and (D16) we see that in most cases the reconnection speed will be given by equation (D13). In other words, as usual, global field line diffusion provides the real constraint on magnetic reconnection in this case.

\section{REFERENCES}

Balbus, S.A. \& Hawley, J.F. 1998, Rev. Mod. Phys., 1, 1

Barghouty, A.F. \& Jokipii, J.R. 1996, ApJ, 470, 858

Barnes, A. 1979, in Solar System Plasma Physics, Vol 1, eds. E.N.Parker, C.F. Kennel \& L.J. Lanzerotti (New York, North-Holland) p. 249

Beck, R., Brandenburg, A., Moss, D., Shukurov, D., \& Sokoloff, D. 1996, ARA\&A, 34, 155 
Biskamp, D. 1984, Phys. Lett. A, 105, 124

1986, Phys. Fluids, 29, 1520

1993, Nonlinear Magnetohydrodynamics, CUP

1996, Astrophys. \& Sp. Sci., 242,165

Biskamp, D., Schwarz, E., \& Drake, J.F. 1997, Phys. Plasmas, 4, 1002

Bisnovatyi-Kogan, G.S. \& Lovelace, R.V.E. 1997, ApJ, 486, L43

Bulanov, S.V., Sakai, J., \& Syrovatskii, S.I. 1979,

Cattaneo, F., \& Vainshtein, S.I. 1991, ApJ, 376, L21

Chang, T. 1998, in AGU Monographs Vol. 104, Geospace Mass and Energy Flow, ed. J.L. Horwitz, D.L. Gallagher, and W.K. Peterson (Washington DC, American Geophysical Union) p. 193

Coleman, P.J. Jr. 1968, ApJ, 153, 371

Cranmer, S., Field, G., \& Kohl, J. 1998, in preparation

Dere, K.P. 1996, ApJ, 472, 864

Diamond, P.H., \& Craddock, G.G. 1990, Comments on the Plasma Physics of Controlled Fusion, 13, 287

Foote, E.A., \& Kulsrud, R.N. 1969, 233, 302

Furth, H.P., Killeen, J., \& Rosenbluth, M.N. 1963, Phys. Fluids, 6, 459 (FKR)

Goldreich, P. \& Sridhar, S. 1995, ApJ, 438, 763 1997, ApJ, 485, 680

Gruzinov, A.V. 1998, ApJ, 501, 787

Gruzinov, A.V., \& Diamond, P.H. 1994, Phys. Rev. Lett., 72(11), 1651

Innes, D.E., Inhester, B., Axford, W.I., \& Wilhelm, K. 1997, Nature, 386, 811

Iroshnikov, P.S. 1963, AZh, 40, 742

Kazantsev, A.P. 1968, Soviet Phys., JETP, 26, 1031 
Kohl, J.L., Noci, G., Antonucci, E., Tondello, G., Huber, M. C. E., Cranmer, S.R., Strachan, L., Panasyuk, A.V., Gardner, L.D., Romoli, M., Fineshi, S., Dobrzycka, D., Raymond, J.C., Nicolosi, P., Seigmund, O.H., Spadaro, D., Benna, C., Ciaravella, A., Giordano, S., Habbal, S.R., Karovska, M., Li, X., Martin, R., Michels, J.G., Modigliani, A., Naletto, G., O’Neal, R.H., Pernechele, C., Poletto, G., Smith, P.L., \& Suleiman, R. 1998, ApJ, 501, L127

Kraichnan, R.H. 1965, Phys. Fluids, 8, 1385

Krause, F., \& Radler, K.H. 1980, Mean-Field Magnetohydrodynamics and Dynamo Theory (Oxford: Pergamon Press)

Kulsrud, R.M., \& Anderson, S.W. 1992, ApJ, 396, 606

Kulsrud, R.M., \& Hahm, T.S. 1982, Physica Scripta, T2/2, 525

Kulsrud, R.M., R. Cen, J.P. Ostriker, \& D. Ryu 1997, ApJ, 480, 481

Lazarian, A. 1992, A\&A, 264, 326 1995, A\&A, 293, 507

Lazarian A., \& Vishniac, E.T. 1996, in Polarimetry of the Interstellar Medium, eds. W.G. Roberge and D.C.B. Whittet, ASP 97, 537

Lazarian, A. \& Pogosyan, D. 1999, ApJ, submitted 1999, ApJ, in preparation

Leamon, R.J., Smith, C.W., Ness, N.F., \& Matthaeus, W.H. 1998, J. Geophys. Res: Space Physics, 103, 4775

Lubow, S.H., \& Pringle, J.E. 1996, MNRAS, 279, 1251

Ma, Z.W., \& Bhattacharjee, A. 1996, Geophys. Research Lett. v. 23, No. 21, 2955

Matthaeus, W.H., \& Lamkin, S.L. 1985, Physics of Fluids, v. 29(8), 2513

Matthaeus, W.H., Oughton, S., Ghosh, S., \& Murshed, H. 1998, Phys. Rev. Lett., 81(10), 2056

Moffatt, H.K. 1978, Magnetic Field Generation in Electrically Conducting Fluids (Cambridge: Cambridge University Press)

Montgomery, D., \& Matthaeus, W.H. 1995, ApJ, 447, 706 
Narayan, R., \& Yi, I. 1995, ApJ, 452, 710

Ng, C.S., \& Bhattacharjee, A. 1996, ApJ, 465, 845

Park, W., Monticello, D.A., \& White, R.B. 1984, Phys. Fluids, 27, 137

Parker, E.N. 1957, J. Geophys. Res., 62, 509 1979, Cosmical Magnetic Fields (Oxford: Clarendon Press) 1988, ApJ, 330, 474 1992, ApJ, 401, 137

Petkaki, P., Malara, F. \& Veltri, P. 1998, ApJ, 500, 483

Petschek, H.E. 1964, The Physics of Solar Flares, AAS-NASA Symposium, NASA SP-50 (ed. W.H. Hess), Greenbelt, Maryland, p. 425

Ruzmaikin A.A., Shukurov A.M. \& Sokoloff D.D. 1988, Magnetic fields of Galaxies (Dordrecht: Kluwer) 1989, MNRAS, 241, 1

Shay, M.A., Drake, J.F., Denton, R.E., \& Biskamp, D. 1998, J. Geophys. Res., 103, 9165

Shivamoggi, B.K. 1997, Journal of Plasma Physics, 58, 329

Similon, P.L. \& Sudan, R.N. 1989, ApJ, 336, 442

Sridhar, S. \& Goldreich, P. 1994, ApJ, 432, 612

Sweet, P.A. 1958, in IAU Symp. 6, Electromagnetic Phenomena in Cosmical Plasma, ed. B. Lehnert (New York: Cambridge Univ. Press), 123

Subramanian, K. 1998, MNRAS, submitted

Vainshtein, S.I. 1970, Sov. Phys. - JETP Lett., 31, 87

Vainshtein, S.I. \& Zel'dovich, Ya.B. 1972, Sov. Phys. - Uspekhi, 15, 159

Van Hoven, G., \& Cross, M.A. 1971, Phys. Fluids, 14, 1141

Vishniac, E.T. 1995a, ApJ, 446, 724 1995b, ApJ, 451, 816 
Vishniac, E.T., \& Lazarian, A. 1998, ApJ, in press 1998b, ApJ, submitted

Zel'dovich, Ya.B., Ruzmaikin, A.A., \& Sokoloff, D.D. 1983, Magnetic Fields in Astrophysics (New York: Gordon and Breach)

Wang, X., Ma, Z.W., \& Bhattacharjee, A. 1992, Phys. Fluids, B 4, 1795 1996, Physics of Plasmas, 3(5), 2129

This preprint was prepared with the AAS IATEX macros v4.0. 


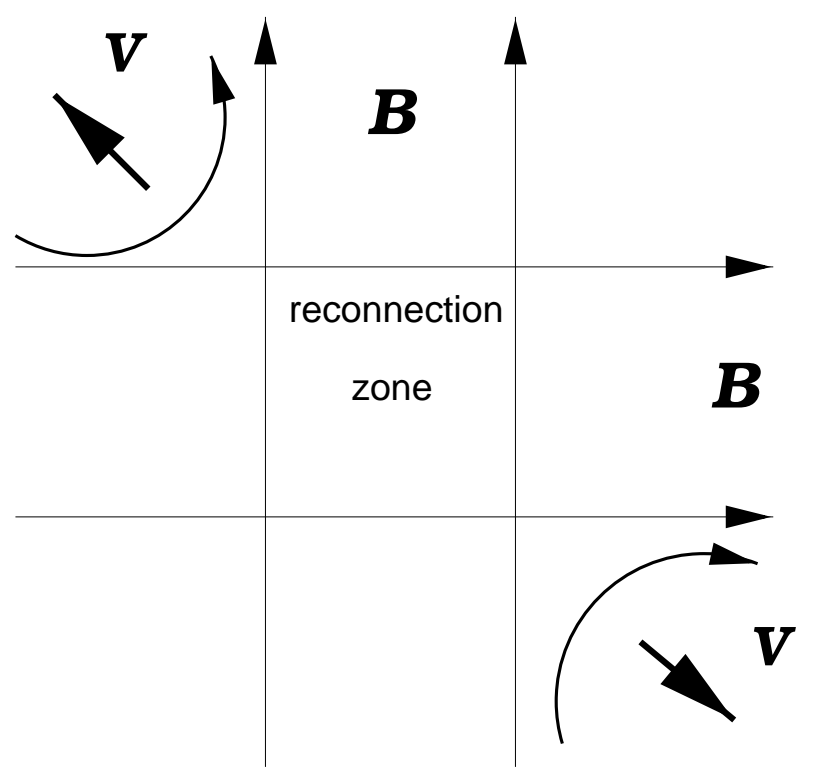

Fig. 1.- The geometry of magnetic field lines in 3D reconnection. The reconnected lines stretch and carry the conducting plasma with them. The plasma is also redistributed along the field lines. 

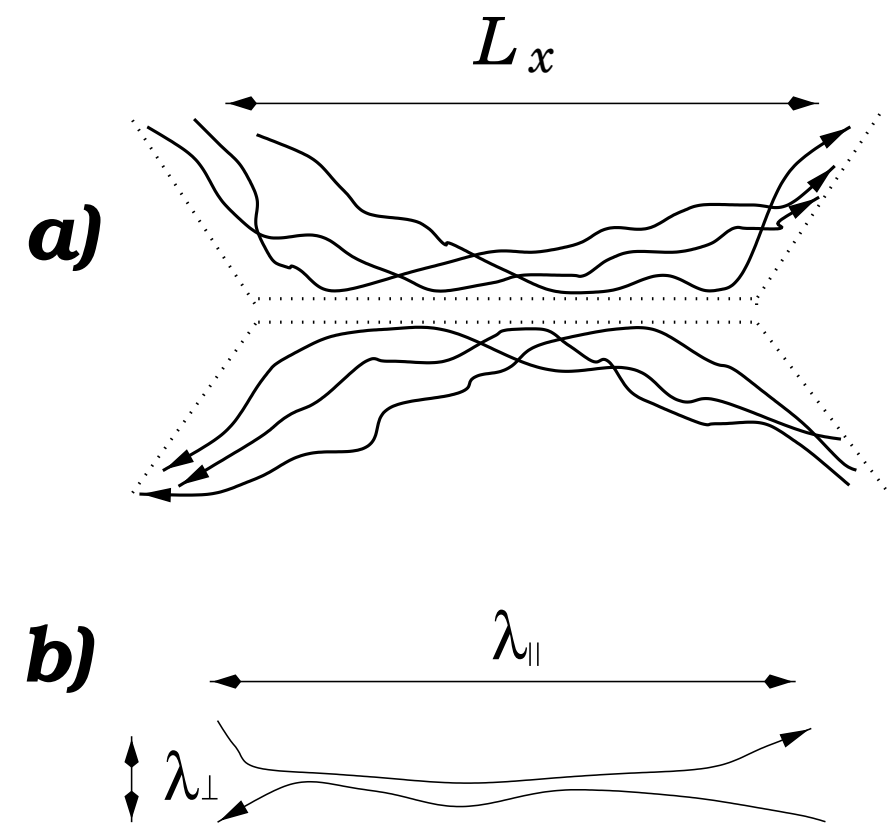

Fig. 2.- a) The structure of the reconnection region when the field is turbulent. Local reconnection events happen on the small scale $\lambda_{\|}$rather than $L_{x}$ and this accelerates reconnection. The plasma is redistributed along the field lines in a layer of thickness $\left\langle y^{2}\right\rangle^{1 / 2}$, which is much thicker than the region $\sim \lambda_{\perp}$ from which the ejection of the magnetic field takes place. b) Local structure of magnetic field lines. 